\title{
A Two-Dimensional Logic for Two Paradoxes of Deontic Modality*
}

\author{
Melissa Fusco \\ Alexander W. Kocurek \\ August 2020. Forthcoming in The Review of Symbolic Logic
}

\begin{abstract}
In this paper, we axiomatize the deontic logic in Fusco 2015, which uses a Stalnaker-inspired account of diagonal acceptance and a two-dimensional account of disjunction to treat Ross's Paradox and the Puzzle of Free Choice Permission. On this account, disjunction-involving validities are a priori rather than necessary. We show how to axiomatize two-dimensional disjunction so that the introduction/elimination rules for boolean disjunction can be viewed as one-dimensional projections of more general two-dimensional rules. These completeness results help make explicit the restrictions Fusco's account must place on free-choice inferences. They are also of independent interest, as they raise difficult questions about how to 'lift' a Kripke frame for a onedimensional modal logic into two dimensions.
\end{abstract}

\section{Introduction}

The validity of or -introduction seems to be a basic rule of natural language disjunction: from $\phi$, one may infer $\phi$ OR $\psi$.

Or-Intro. $\phi \models(\phi$ OR $\psi)$

Yet the rule apparently fails in the scope of deontic modals. For example, the nonentailment known as Ross's Puzzle illustrates the failure of Or-Intro in the scope of 'ought' (henceforth O) (Ross, 1941):

Ross's Puzzle. $O \phi \not \models O(\phi$ OR $\psi)$

*Many thanks to Peter Fritz, Lloyd Humberstone, and an anonymous reviewer for their helpful feedback. Thanks also to the audience at the 2020 Philosophical Applications of Modal Logic conference. The authors contributed equally to this paper and are listed in alphabetical order. 
As an illustration, observe that (1-a), famously, does not seem to entail (1-b):

(1) a. You ought to post the letter.

b. You ought to post the letter or burn it.

Similarly, Or-Intro does not seem valid in the scope of 'may' (henceforth $M$ ). For instance, (2-a) does not seem to entail (2-b):

a. You may post the letter.

b. You may post the letter or burn it.

An attractive explanation for this is that (2-b) seems to entail that both disjuncts are permissible —an observation known as Free Choice Permission (Kamp, 1973):

FCP. $M(\phi$ OR $\psi) \models M \phi \wedge M \psi$

For example, the inference from (3-a) to (3-b) and (3-c) seems valid:

(3) a. You may have an apple or a pear.

b. You may have an apple.

c. You may have a pear.

This would explain why (2-a) doesn't entail (2-b): if it did, then (2-a) would entail you may burn the letter, which is not the case! ${ }^{1}$

It is well-known that Ross's Puzzle and FCP cause trouble for standard deontic logic, which interprets $O$ as a normal modal operator, with $M$ as its dual. As a consequence, these operators are monotonic: if $\phi$ entails $\psi$, then $O \phi$ entails $O \psi$, and $M \phi$ entails $M \psi$. This means that standard deontic logic predicts that (i) $O \phi$ entails $O(\phi$ OR $\psi)$ and $M \phi$ entails $M(\phi$ OR $\psi)$, and (ii) if FCP holds, then $M \phi$ entails $M \psi$ for any $\phi$ and $\psi$. These predictions reveal a deep problem at the foundations of standard deontic logic.

Ross's Puzzle is problematic for any deontic logic that validates two key principles:

Necessitation. If $\models \phi$, then $\models O \phi$

K Axiom. $\models O(\phi \rightarrow \psi) \rightarrow(O \phi \rightarrow O \psi)$

${ }^{1}$ Note that while (3-a) seems to entail (3-b) and (3-c), it resoundingly fails to entail (i):

(i) You may have an apple and a pear.

This additional datum is sometimes called 'exclusivity'.

EX. $M(\phi$ OR $\psi) \not \models M(\phi \wedge \psi)$

See, inter alia, Simons 2005, Fox 2007, and Barker 2010. See also Fusco 2020a for empirical work on the nature of this exclusivity. 
From these principles (together with classical logic), we can derive $O \phi \rightarrow O(\phi$ or $\psi)$. Here is the proof:

1. $\phi \rightarrow(\phi$ OR $\psi)$

Or-Intro

2. $O(\phi \rightarrow(\phi$ OR $\psi))$

Necessitation, 1

3. $O(\phi \rightarrow(\phi$ OR $\psi)) \rightarrow(O \phi \rightarrow O(\phi$ OR $\psi))$

K Axiom

4. $O \phi \rightarrow O(\phi$ OR $\psi)$

modus ponens, 2,3

Thus, if one wishes to capture Ross's Puzzle in a deontic logic, one seems committed either to rejecting Necessitation or rejecting the K Axiom. But which is the culprit, and why?

Fusco (2015) suggests that the culprit is Necessitation. To see why, let's consider why the inference from (1-a) to (1-b) seems bad. One reason it seems bad to say you ought to post the letter or burn it is that this seems to imply that both disjuncts are permissible. The following inference pattern-Free Choice Obligation-seems intuitively valid:

FCO. $O(\phi$ or $\psi) \models M \phi \wedge M \psi$

Thus, to illustrate, (4-a) seems to entail (4-b) and (4-c):

(4) a. You ought to either do the dishes or take out the trash.

b. You may do the dishes.

c. You may take out the trash.

Moreover, FCO follows from FCP together with a relatively plausible principle relating 'ought' and 'may':

Ought Implies May. $O \phi \models M \phi$

But if FCO holds, then already Necessitation is enough to wreak havoc: Necessitation plus FCO entail that everything is permissible: ${ }^{2}$

1. $(\phi$ OR $\neg \phi)$

tautology

2. $O(\phi$ OR $\neg \phi)$

Necessitation, 1

3. $M \phi \wedge M \neg \phi$

$\mathrm{FCO}, 2$

This provides one motivation for thinking that the way forward for deontic logic is to reject Necessitation.

Two-dimensional semantics offers an elegant way of developing Necessitationfree modal logics: while (5-a) below is "necessary" in the sense of being knowable a priori, it is not metaphysically necessary, in that things could have been different from how they actually are (Crossley and Humberstone, 1977; Davies and Humberstone, 1980; Kaplan, 1989).

\footnotetext{
${ }^{2}$ Note it does not help here to restrict FCO to $\phi$ and $\psi$ that are possible; that still would imply that for any contingent $\phi$ is permissible.
} 
a. Everything is as it actually is.

b. Necessarily, everything is as it actually is.

Perhaps, then, the key to solving these puzzles is to move to a two-dimensional framework. This is the strategy pursued by Fusco (2015).

The main idea behind Fusco's semantics is to introduce a two-dimensional entry for ' OR ' on which it behaves classically in unembedded contexts but nonclassically under the scope of deontic modals. However, while this semantics captures Ross's Puzzle and FCP, there still remains a further question regarding what the complete logic of her semantics is.

In this paper, we answer this question. We show how to axiomatize the logic of two-dimensional disjunction so that the introduction/elimination rules for boolean disjunction can be viewed as one-dimensional projections of more general rules. In addition, we prove several soundness and completeness results for two-dimensional deontic logics, which help make explicit the background assumptions and scope of Fusco's account.

We take these completeness results to be of independent interest, as they raise interesting questions about how to "lift" a Kripke frame for a one-dimensional modal logic into two dimensions. These issues arise especially in the context of the picture of communication from Stalnaker 1978, where diagonalization plays a key role. In particular, two-dimensionalizing deontic logic in a Stalnakerian framework seems to require an interesting and substantive metaphysical assumption about the nature of deontic accessibility, viz., that which worlds are deontically accessible does not vary from world-to-world. ${ }^{3}$

Here is a brief outline. In $\S 2$, we review the philosophical motivations, formal semantics, and logic of standard two-dimensionalism. In $\S 3$, we present Fusco's two-dimensional semantics for disjunction and articulate different ways to axiomatize the logic of disjunction prior to adding deontic modals. In $\S 4$, we extend two-dimensionalism with deontic modals and show how doing so can capture both Ross's Puzzle and FCP. The details of the completeness proofs are given in the technical appendices.

\section{A Crash Course in Two-Dimensionalism}

\subsection{Two Notions of Necessity and Two Notions of Consequence}

In two-dimensional semantics, the truth of a well-formed formula $\phi$ in a model $\mathcal{M}$ is relativized to two parameters. The first, the world-as-actual, plays a role in

\footnotetext{
${ }^{3}$ This bears resemblance to a point made by Hawthorne and Magidor (2009) about epistemic accessibility.
} 
determining the propositional content expressed by $\phi .{ }^{4}$ The second, the world of evaluation, plays the role of the world in which that proposition's truth-value is interrogated; it is also the only parameter shifted by the standard modal operators $\square$ and $\diamond$. Two basic two-dimensional operators, @ (usually glossed as 'actually') and $\dagger$, can be added alongside $\square$ and $\diamond: 5$

(口) $y, x \Vdash \square \phi$ iff for all $x^{\prime}: y, x^{\prime} \Vdash \phi$

(@) $y, x \Vdash @$ iff $y, y \Vdash \phi$

(†) $y, x \Vdash \dagger \phi$ iff $x, x \Vdash \phi$

One motivation for moving to a two-dimensional framework is the fact that we can regiment several philosophically important distinctions in a unified way (Davies and Humberstone, 1980). To illustrate, recall (5-a) and (5-b) from $\S 1$ :

a. Everything is as it actually is.

b. Necessarily, everything is as it actually is.

We observed that while (5-a) seems to be a logical truth, it does not seem to be necessarily true, i.e., (5-b) seems false. The problem is that we cannot accept (5-a) as a logical truth in a normal modal logic while denying (5-b). Let $\bowtie$ stand for $\ulcorner$ Everything is as it actually is $\urcorner .{ }^{6}$ In the two-dimensional framework, we can analyze this sentence as follows: ${ }^{7}$

$(\bowtie) y, x \Vdash \bowtie$ iff $y=x$

Then we have the following argument for $(5-b)$ :

1. $\bowtie$ is a logical truth

2. Necessitation holds, i.e., if $\phi$ is a logical truth, then so is $\square \phi$.

3. $\square \bowtie$ is a logical truth.

\footnotetext{
${ }^{4} \mathrm{We}$ follow the literature here in defining a proposition to be a function from worlds to truthvalues.

${ }^{5}$ Technically, the satisfaction relation $\Vdash$ should always be relativized to a model $\mathcal{M}$. In our informal exposition, however, we will often drop mention of the model for readability.

${ }^{6}$ For similar sentential constants, compare (for "floating" actuality) 'I' from Humberstone (2004, pg. 54), and (for "anchored" actuality) the constant ' $n$ ' in Meredith and Prior (1965). In a language with propositional quantifiers and @, we could express $\bowtie$ as $\forall p(@ p \rightarrow p)$.

${ }^{7}$ These truth conditions ignore complications that arise when $x$ and $y$ are qualitative duplicate worlds. We set this complication aside.
} 
If we want to deny the conclusion, we need to reject one of the premises. But which?

Two-dimensionalism provides us with an elegant answer: which premise you should give up depends on your notion of consequence. First, let us say an argument is strictly valid just in case it preserves truth over all points of evaluation, i.e., all pairs of worlds $\langle y, x\rangle$. Strict validity captures the thought that a valid argument is meant to preserve truth in every possible scenario. On this notion of logical consequence, Necessitation still holds: if $\phi$ is true at every point of evaluation $\langle y, x\rangle$, then so is $\square \phi$. However, the first premise of the argument for $(5-b)$ is false, since there are non-diagonal points where the $x$ - and $y$-coordinates do not agree on what is the case-that is, whatever world is actual, it still could have been that things were different from how they actually are. Since $\bowtie$ only holds on diagonal points, it is not a strict logical truth.

However, $\bowtie$ does hold on all diagonal points. An argument is diagonally valid just in case it preserves truth over all diagonal points, i.e., all pairs of the form $\langle z, z\rangle$. This captures the thought that a valid argument is meant to preserve truth no matter which world is actual. On this notion of logical consequence, the first premise of the argument for (5-b) is true, but the second premise is false-e.g., $\bowtie$ is diagonally valid, but $\square \bowtie$ is not. In general, whenever something is diagonally but not strictly valid, we'll have a counterexample to Necessitation.

Quantification over diagonal points also allows one to represent the difference between metaphysical necessity and a priority in a two-dimensional framework. This is just the difference between $\phi$ 's being "horizontally" valid, i.e., holding everywhere along the $x$-axis, and $\phi$ 's being diagonally valid. ${ }^{8}$ That is, fixing $y$ as the actual world, $\phi$ is metaphysically necessary iff it holds at $\langle y, x\rangle$ for all $x$, whereas $\phi$ is a priori iff it holds at $\langle z, z\rangle$ for all $z .^{9}$ The latter notion can be represented by introducing a new defined operator for a priori truth: $A \phi:=\square \dagger \phi$. It is easy to check that $A$ has the following truth conditions:

(A) $y, x \Vdash A \phi$ iff for all $z: z, z \Vdash \phi$.

While $\bowtie$ ('everything is as it actually is') is not metaphysically necessary, it is a priori: that is, $A \bowtie$ is a strict logical truth. In fact, even though Necessitation does not hold for diagonal validity, the following variant of Necessitation does:

A Priorization. If $\models \phi$, then $\models A \phi$.

That is: if $\phi$ is a diagonal logical truth, then so is $A \phi$. Strict validity is a formalization of necessary entailment, while diagonal validity is a formalization of a priori entailment.

\footnotetext{
${ }^{8}$ See Davies and Humberstone 1980; Kaplan 1989. For classic examples where metaphysical necessity comes apart from a priority, see Kripke 1980; Kaplan 1989.

${ }^{9}$ This idea can be found, inter alia, in van Fraassen (1977) and Lewis (1973, §2.8), who in turn cites Kamp (1971) and Vlach (1973). See also Chalmers (2004).
} 


\subsection{Stalnaker's Two Dimensionalism}

Another classic application of two-dimensionalism, which will provide us with a metalanguage (rather than object language) gloss on A, comes from Stalnaker 1978. There, Stalnaker uses two-dimensionalism to model conversations in which speakers informatively assert sentences whose propositional content is under threat from indeterminacy. To clarify the problem, he introduces the notion of a context set: a set of possibile worlds left open by what is accepted in common by the interlocutors of a conversation. Conversational update on an assertion of $\phi$ is a quasi-Bayesian procedure which aims to eliminate from the context set any worlds which are incompatible with $\phi$ 's content.

Suppose, however, that it is unknown-or perhaps, in the case of the open future, even metaphysically indeterminate-which world is actual. Then there will be cases in which it is unknown or indeterminate what the content of an assertion of $\phi$ is, and thus unclear which worlds should be "thrown out" of the context set if the assertion is to be accepted. Stalnaker's flagship example involves the word 'you' in the asserted sentence $\ulcorner$ You are a fool $\urcorner$ (pg. 81). If it is unknown, or indeterminate, who the addressee of the context is, it will be unknown, or indeterminate, which person is being claimed to be a fool, and so it will be unknown or indeterminate which update is being proposed.

Following Stalnaker, we can visualize the situation using two-dimensional matrices. In these matrices, the rows represent the world-as-actual ( $y$-coordinate)-a role which contributes to determining the proposition expressed by a sentence (e.g., determining the referent of 'you')—while the columns represent the world of evaluation ( $x$-coordinate), where the content, once determined, is assessed. If the content of a sentence depends on which world is actual, then the rows of the matrix will not be identical.

So to use Stalnaker's example, suppose the speaker is addressing O'Leary in worlds $i$ and $j$ and to Daniels in world $k$, and moreover $\mathrm{O}^{\prime}$ Leary is a fool in worlds $i$ and $k$ but not in world $j$, whereas Daniels is a fool only in $j$. In that case, we can represent the distribution of truth values of $\ulcorner$ You are a fool $\urcorner$ as in Figure 1. For instance, the cell on row $i$ and column $j$ contains the truth value of $\ulcorner$ You are a fool $\urcorner$ at the point $\langle i, j\rangle$, which is true iff the person that the speaker is addressing in world $i$ is a fool in world $j$. Since at world $i$, the speaker is addressing $\mathrm{O}^{\prime}$ Leary, and since $\mathrm{O}^{\prime}$ Leary is not a fool at world $j,\ulcorner$ You are a fool $\urcorner$ is false at $\langle i, j\rangle$. Which worlds need to be thrown out of the context set upon accepting an assertion of $\ulcorner$ You are a fool $\urcorner$ depends on which world is actual: if $i$ or $j$ is actual, we throw out $j$; if $k$ is actual, we throw out $i$ and $k$. But-to reiterate the problem Stalnaker is raising - which world is actual is exactly what is either unknown or indeterminate in context (op. cit., pg. 90).

In such a predicament, Stalnaker proposes that a sentence $\phi$ can be rationally (re)interpreted as $\dagger \phi$. As Stalnaker puts it, this move, called diagonalization, 


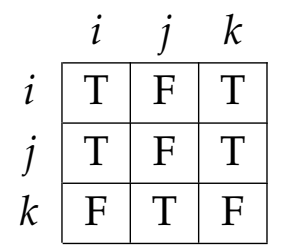

$\ulcorner$ You are a fool $\urcorner$

Figure 1: A two-dimensional matrix for $\ulcorner$ You are a fool $\urcorner$ (Stalnaker, 1978, pg. 81).

interprets $\phi$ as something like "what $\phi$ says is true", where "what $\phi$ says" is the proposition expressed by $\phi$ given a world as actual (pg. 82). ${ }^{10}$ This is visualized in Figure 2. Diagonalization restores a uniformity condition to matrices: the same proposition is expressed relative to each candidate for actuality. ${ }^{11}$ In a scenario illustrated by Figure 1, updating the context set with the diagonalized assertion amounts to eliminating worlds $j$ and $k$ from the context set.

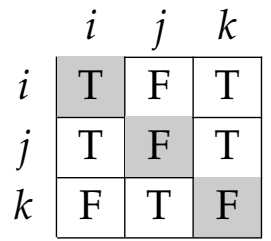

$\ulcorner$ You are a fool $\urcorner$

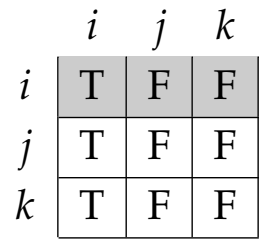

$\ulcorner\nmid$ You are a fool $\urcorner$

Figure 2: Visualizing diagonalization using matrices.

Stalnaker's theory of assertion reveals a further distinction in the notion of logical consequence worth highlighting. In $\S 2.1$, we introduced a classical notion of consequence, which defines validity in terms of preservation of truth: an argument is valid in this sense if the conclusion is true at any point of evaluation where the premises are true. But there is also an informational notion of consequence, which defines validity in terms of preservation of acceptance. An argument is valid in this sense if the conclusion is accepted at any context set where the premises are accepted. ${ }^{12}$ In a static one-dimensional framework, acceptance can be mod-

\footnotetext{
${ }^{10}$ For a suggestion that diagonalization can also occur in the scope of attitude verbs like 'believes', see Stalnaker (1981).

${ }^{11}$ In the literature, Hawthorne and Magidor (2009) object that diagonalization does not restore uniformity unless certain tendentious conditions on knowledge are met: namely, that the same worlds horizontally accessible on each row. We bracket this concern here, particularly because our discussion is applicable to nonepistemic as well as epistemic indeterminacy.

${ }^{12}$ For more on this notion, see Stalnaker 1975's notion of reasonable inference, as well as, inter
} 
eled as global truth, i.e., truth relative to every world in a context set. Thus, $\Gamma$ informationally entails $\phi$ whenever $\square \Gamma:=\{\square \gamma \mid \gamma \in \Gamma\}$ classically entails $\square \phi .{ }^{13}$

$$
\begin{array}{lll}
\Gamma \text { classically entails } \phi & :=\forall x:(x \Vdash \Gamma \Rightarrow x \Vdash \phi) \\
\Gamma \text { informationally entails } \phi & :=(\forall x: x \Vdash \Gamma) \Rightarrow(\forall x: x \Vdash \phi)
\end{array}
$$

But what about diagonalization? In Stalnaker's two-dimensional framework, diagonalization may occur before acceptance into the common ground. ${ }^{14}$ Hence there is a strict-diagonal distinction to be had at the level of informational consequence as well as classical consequence. Informational strict consequence can be glossed like this: no matter which world is actual, if the content of the premises were accepted, so would the content of the conclusion. Stated in terms of classical strict entailment: $\Gamma$ informationally strictly entails $\phi$ whenever $\square \Gamma$ classically strictly entails $\square \phi$. Informational diagonal consequence, by contrast, can be glossed like this: if the diagonalized content of the premises are accepted, then so is the diagonalized content of the conclusion. Stated in terms of classical strict entailment: $\Gamma$ informationally diagonally entails $\phi$ whenever $\square \nmid \Gamma:=\{\square \dagger \gamma \mid \gamma \in \Gamma\}$ classically strictly entails $\square \dagger \phi$.

Because $A:=\square \dagger$ can be seen as a priority of the relevant, contextually-situated kind, we will also call informational diagonal consequence a priori consequence, which we will write as ' $\triangle$ '. This notion of consequence matches more closely with the preservation of acceptance in Stalnaker's framework. Moreover, it is a priori consequence which will be key to both validating FCP and blocking Ross's Puzzle on Fusco's semantics: it is a notion of consequence that is just weak enough to validate the former but not the latter. ${ }^{15}$

Thus, we have four notions of consequence-varying along two dimensions,

alia, Veltman 1996; Yalcin 2007; Bledin 2015.

${ }^{13}$ The difference between classical and informational consequence mirrors the distinction between local and global consequence that one finds in the literature on modal logic (Blackburn et al., 2002). For unarticulated 'boxlike' modalities in natural language, it is worth noting Kratzer's influential proposal that bare indicative conditionals contain a covert necessity operator in the consequent Kratzer (1981, 1986).

${ }^{14}$ Indeed, Heim $(2004, \S 9)$ suggests always diagonalizing, noting that diagonalization is superfluous if uniformity is already satisfied.

${ }^{15}$ One may object that a priori consequence is an odd notion of consequence to validate FCP in, since it validates the inference from $p$ to $A p$. It is important to bear in mind, however, that we are using the term "a priori" in a context-specific sense. In this sense, $\phi$ is a priori if, given the current conversational context, it is commonly accepted that $\phi$, where common acceptance is cashed out recursively (everyone in the conversation accepts it, and everyone accepts that everyone excepts it, etc.). Thus, $A \phi$ means something more like $\ulcorner$ it is a priori that $\phi$ given what is commonly accepted in the current conversation $\urcorner$. On that understanding, the inference from $p$ to $A p$ is not surprising: if the speakers all commonly accept $p$, then they commonly accept that they commonly accept $p$. 
strict vs. diagonal and classical vs. informational-illustrated in Figure $3 .{ }^{16} \mathrm{~A}$ priori consequence occupies the lower right-hand side of the relevant space of possibilities.

\begin{tabular}{r|c|c} 
& classical & informational \\
\hline strict & $\Gamma \models \phi$ & $\square \Gamma \models \square \phi$ \\
\hline diagonal & $\dagger \Gamma \models \dagger \phi$ & $\square \dagger \Gamma \models \square \dagger \phi$ \\
& & $\Gamma \triangleright \phi$
\end{tabular}

Figure 3: Different notions of consequence summarized.

\subsection{Axiomatization of Two-Dimensional Semantics}

One of our primary goals in this paper is to give an axiomatization of Fusco's twodimensional extension of deontic logic. So we should first review axiomatizations for ordinary two-dimensional logic before extending them with deontic modals. We give axiomatizations of classical strict consequence, as the other notions of consequence can be defined in terms of classical strict consequence using $\dagger$ and $\square$ (as in Figure 3).

Definition 1. Given a set Prop $=\left\{p_{1}, p_{2}, p_{3}, \ldots\right\}$ of proposition letters, the basic two-dimensional language $\mathcal{L}^{2 \mathrm{D}}$ is defined recursively in Backus-Naur form as follows:

$$
\phi::=\operatorname{Prop}|\neg \phi|(\phi \wedge \phi)|\square \phi| @ \phi \mid \dagger \phi .
$$

$\diamond, \vee, \rightarrow$ and $\leftrightarrow$ defined as usual; in particular, note that $(\phi \vee \psi):=\neg(\neg \phi \wedge \neg \psi)$. We also define $A \phi$ (it is a priori that $\phi$ ) as $\square \dagger \phi$.

Definition 2. A basic matrix frame is a Kripke frame $\left\langle W \times W, R_{\square}, R_{@}, R_{\dagger}\right\rangle$, where $W \neq \varnothing$ and:

- $\langle y, x\rangle R_{\square}\left\langle y^{\prime}, x^{\prime}\right\rangle$ iff $y=y^{\prime}$

${ }^{16}$ Note that in the two-dimensional setting, informational strict consequence does not align neatly with global strict consequence. Global consequence is usually defined in terms of points of evaluation: an argument is globally valid if its conclusion holds at every point of evaluation whenever its premises hold at every point of evaluation. Stated in terms of classical (i.e., local) strict entailment: $\Gamma$ globally strictly entails $\phi$ whenever $\square \dagger \square \Gamma:=\{\square \dagger \square \gamma \mid \gamma \in \Gamma\}$ classically strictly entails $\square \dagger \square \phi$. This is not the same as informational strict consequence, since the world-as-actual is held fixed from premises to conclusion. Thus, informational strict consequence is stronger than global strict consequence. On the other hand, informational diagonal consequence and global diagonal consequence do coincide. 
- $\langle y, x\rangle R_{\Theta}\left\langle y^{\prime}, x^{\prime}\right\rangle$ iff $y^{\prime}=x^{\prime}=y$.

- $\langle y, x\rangle R_{\dagger}\left\langle y^{\prime}, x^{\prime}\right\rangle$ iff $y^{\prime}=x^{\prime}=x$.

Let $\mathrm{M}$ be the class of basic matrix frames. A basic matrix model is a pair of a basic matrix frame $\left\langle W \times W, R_{\square}, R_{\Theta}, R_{\dagger}\right\rangle$ and a valuation function $V$ : Prop $\rightarrow \wp(W \times W)$.

We define the satisfaction relation $\Vdash$ between a pointed matrix model $\mathcal{M}, y, x$ and a $\mathcal{L}^{2 \mathrm{D}}$-formula $\phi$ in the usual manner for the Kripke semantics. Where $\Gamma$ is a set of formulas, we'll write $\mathcal{M}, y, x \Vdash \Gamma$ to mean that $\mathcal{M}, y, x \Vdash \gamma$ for all $\gamma \in \Gamma$. Finally, we define classical strict consequence: where $\Gamma$ is a set of $\mathcal{L}^{2 \mathrm{D}}$-formulas and $\phi$ is a $\mathcal{L}^{2 \mathrm{D}}$-formula, $\Gamma \models_{2 \mathrm{D}} \phi$ iff for all pointed basic matrix models $\mathcal{M}, y, x$, if $\mathcal{M}, y, x \Vdash \Gamma$, then $\mathcal{M}, y, x \Vdash \phi$.

Definition 3. Let 2D be the Hilbert-style proof system consisting of the following axioms and rules: (i) all propositional tautologies as axioms; (ii) the rules of Modus Ponens and Uniform Substitution; (iii) the K Axiom and Necessitation for each primitive modal operator; and (iv) the following axioms:

$$
\begin{array}{ll}
T_{\square} \quad \square p \rightarrow p & R_{\dagger} \quad \dagger p \leftrightarrow \neg \neg p \\
5_{\square} \diamond p \rightarrow \square \diamond p & X_{\rightarrow} \quad \dagger(p \rightarrow @ p) \\
@ 5 \diamond \quad \diamond @ p \rightarrow @ p & Y_{\rightarrow} \quad @(p \rightarrow \dagger p) \\
G \quad \square p \rightarrow @ p & 4_{A} \quad \square \dagger p \rightarrow \square \dagger \square \dagger p \\
R_{@} @ p \leftrightarrow \neg @ \neg p & 5_{A} \quad \diamond \dagger p \rightarrow \square \dagger \diamond \dagger p
\end{array}
$$

We write $\Gamma \vdash_{2 D} \phi$ if for some $\gamma_{1}, \ldots, \gamma_{n} \in \Gamma$, the formula $\left(\gamma_{1} \wedge \cdots \wedge \gamma_{n}\right) \rightarrow \phi$ is derivable in $\mathbf{2 D}$.

The following is proven in Fusco 2020b.

Theorem 1. 2D is sound and complete for classical strict consequence over $M$.

A priori consequence can then just be defined in terms of classical strict consequence: $\Gamma \triangleright_{2 D} \phi$ iff for some $\gamma_{1}, \ldots, \gamma_{n} \in \Gamma: \vdash_{2 D}\left(A \gamma_{1} \wedge \cdots \wedge A \gamma_{n}\right) \rightarrow A \phi$.

\section{4 Åqvist logic}

The matrix semantics given above is more general than the usual presentation of two-dimensionalism in at least one respect: we allow atomics to represent arbitrary matrices like the one provided for $\ulcorner$ You are a fool $\urcorner$, whose content varies with the world-as-actual.

By contrast, according to one common strand of two-dimensionalism, valuation functions interpret atomics as sets of worlds, not sets of pairs of worlds. This means 
the truth of atomics never depends on the world-as-actual parameter. These "standard" truth conditions build in the requirement that the atomics are reserved for sentences that satisfy the Stalnakerian uniformity condition introduced in $\S 2.2$, i.e., that $\langle y, x\rangle \in V(p)$ iff $\left\langle y^{\prime}, x\right\rangle \in V(p)$ for any $x, y, y^{\prime}$. For these sentences, necessity and a priority coincide. ${ }^{17}$

It is natural to ask how imposing this restriction on the valuations of atomics affects the logic given in $\S 2.3$. We call such logics Åqvist logics (Åqvist, 1973; Segerberg, 1973). It turns out that their distinguishing axiom is $\AA$ :

$\AA \quad \alpha \leftrightarrow \dagger \alpha$ for any atomic $\alpha$ (Segerberg, 1973, pg. 95)

In terms of matrices, $\AA$ says that rows of the matrix of an atomic must be duplicates, i.e., each column must either be a column of Ts or a column of Fs.

Definition 4. A matrix model $\left\langle W \times W, R_{\square}, R_{\Theta}, R_{\dagger}, V\right\rangle$ is Åqvist iff for all $x, y, y^{\prime} \in$ $W$ :

$$
\langle y, x\rangle \in V(p) \Leftrightarrow\left\langle y^{\prime}, x\right\rangle \in V(p) .
$$

Let $M \AA$ be the class of Åqvist matrix models.

Note that we can define an "Åqvist operator" $\AA$ which applies to a formula $\phi$ iff $\phi$ 's truth is insensitive to the world-as-actual parameter, i.e., iff it is interpreted the same way on every row:

$$
\AA \phi \quad:=\square \dagger \square(\phi \leftrightarrow \dagger \phi)
$$

Then we could reformulate $\AA$ as the axiom schema $\AA \alpha$ for any atomic $\alpha$. The $\AA$ operator turns out to be important for Fusco's explanation of FCP, as the inference only holds in her semantics for $\phi$ and $\psi$ that have this "uniformity" feature.

As Segerberg is at pains to emphasize, an Åqvist logic is not, in general, closed under uniform substitution. ${ }^{18}$ Rather, it is closed only under substitution within the 1D fragment of the language. ${ }^{19}$ Thus, in order to axiomatize Åqvist logics, we need to formulate proof systems that are not closed under Uniform Substitution, but still allow Uniform Substitution when no appeal to $\AA$ has been made. This is achieved as follows:

${ }^{17}$ One can compare here the PQTI sentences of Chalmers (2012).

${ }^{18}$ For discussion, see, inter alia, Smiley 1982; Holliday et al. 2013.

${ }^{19}$ This limitation on closure under substitution follows the spirit of, e.g., Williamson (2013), who remarks that "the obvious rationale for insisting on ... closure... under uniform substitution in a propositional system is a reading of non-logical sentence letters as propositional variables" (Williamson, 2013, pg. 76, emphasis added; see also Burgess, 1999, pg. 176). Similarly, in a 2D framework Gregory (2001) remarks that "Good modal [arguments] —at least one variety—are ones which are informally sound...[meaning that] for any interpretation of $\mathcal{L}$, the propositions assigned to [the premises] entail the proposition assigned to [the conclusion] (op. cit., pg. 58, emphasis added). This restriction on closure will exclude arbitrary dipropositions, like $\bowtie$ and the (undiagonalized) $\ulcorner$ You are a fool $\urcorner$, from the relevant standard of good argument. 
Definition 5. Let 2DÅ be the Hilbert-style proof system consisting of Modus Ponens, Necessitation for each primitive modal operator, $\AA$, and the axiom schema:

$2 D \phi$, where $\phi$ is a theorem of $2 \mathrm{D}$.

In 2DÅ, we can appeal to Uniform substitution in deriving an ordinary theorem of 2D. But once we start reasoning with the consequences of $\AA$, we are no longer allowed to appeal to Uniform Substitution and must instead rely on the other rules and axioms. The following is proven in Fusco 2020b:

Theorem 2. 2DÅ is sound and complete for strict classical consequence over MÅ.

\section{Two-Dimensional Disjunction}

\subsection{An Actuality-sensitive Semantics for Disjunction}

We now return to Fusco's strategy for solving the deontic puzzles sketched in $\S 1$. According to this two-dimensional strategy, what we need for Ross's Puzzle and FCP is a semantics for disjunction where Or-Intro is a priori, but not necessary. In this section, we review Fusco's proposal and explain how an axiomatization for the basic two-dimensional language can be extended to include her two-dimensional disjunction.

This semantics is motivated by Groenendijk and Stokhof (1982) and Lewis (1982)'s two-dimensional approach to "whether $p$ or $q$ " attitude ascriptions, as in

(6) Bob knows [whether [ $p$ or $q]$.

(7) Bob doesn't know [whether [ $p$ or $q]$ ].

The insight here is that (6) and (7) credit Bob with knowledge and ignorance, respectively, of the actually true answer to the question $\ulcorner p$, or $q$ ? ? . According to Groenendijk \& Stokhof and Lewis, $\ulcorner$ whether $p$ or $q\urcorner$ is a two-dimensional propositional concept, which expresses the proposition that $p$, if $p$ rather than $q$ is true in the world-as-actual, and $q$, if $q$ rather than $p$ is true in the world-as-actual. Thus a speaker of (6) or (7) can credit Bob with knowledge (or ignorance) of this proposition, even if he himself does not know it.

Building off this, Fusco (2015) proposes we interpret bare disjunction along similar lines: if $p$ but not $q$ is actually true, then $p$ or $q$ expresses the proposition that $p$; and if $q$ but not $p$ is actually true, then $p$ or $q$ expresses the proposition that $q$. The key difference between the semantic entries for $\ulcorner$ whether $p$ or $q\urcorner$ above and Fusco's semantics for $p$ or $q$ concerns the cases where either both disjuncts true or neither are: in that case, for Fusco, $p$ OR $q$ just expresses the ordinary boolean 
disjunction $p \vee q .{ }^{20}$

We can state this semantics for or in terms of a simpified answerhood operator (Dayal, 1996, 2016). This operator takes and world $w$ and disjuncts $\phi$ and $\psi$ as arguments and outputs the true-in- $w$ answers to the question of whether $\phi$ or $\psi$, if there are any. Otherwise, it outputs both answers:

$$
\operatorname{Ans}(w, \phi, \psi)= \begin{cases}\{\phi\} & \text { if } w, w, \Vdash \phi \text { and } w, w, \Vdash \psi \\ \{\psi\} & \text { if } w, w, \Downarrow \phi \text { and } w, w, \Vdash \psi \\ \{\phi, \psi\} & \text { otherwise. }\end{cases}
$$

The truth conditions for disjunction can then be given as follows:

( oR ) $y, x \models(\phi$ oR $\psi)$ iff $\exists \alpha \in \operatorname{Ans}(y, \phi, \psi): y, x \Vdash \alpha$.

Figure 4 illustrates these truth conditions in matrix form. Notice that or is, as Humberstone (2020, §4.7) puts it, a kind of two-dimensional isotope of boolean $\vee$-that is, OR and $\vee$ are equivalent along the diagonal. Thus, for diagonal consequence, all the standard rules governing boolean disjunction hold of oR . But off the diagonal, or and $\vee$ come apart. For example, in Figure $4, w_{2}, w_{3} \forall p$ OR $q$ even though $w_{2}, w_{3} \Vdash p$.

On this picture of disjunction, $p$ OR $\neg p$ is like $\bowtie$, in that it is an a priori truth that cannot be necessitated. (Indeed, if there are only finitely many atomics $p_{1}, \ldots, p_{n}$ and all the rows of the truth table are represented by a unique world, then $\bigwedge_{i}\left(p_{i}\right.$ OR $\left.\neg p_{i}\right)$ generates the same matrix as $\bowtie$.) The feature of disjunction will be responsible for nonclassical behavior in the scope of deontic modals ( $\$ 4$ ).

Note also that ( $\phi$ or $\psi$ ) will generally not be Aqquist, even if both $\phi$ and $\psi$ are. Figure 5 shows how two-dimensional disjunction, like the @ operator itself, can build non-Åqvist matrices out of the matrices for Åqvist atomics. The diagonals of these matrices for disjunction reflect the classical profile of $\vee$ : the more disjuncts a classical disjunction has, the more states in a classical (viz., one-dimensional) truth table it is true in.

${ }^{20}$ Groenendijk \& Stokhof's entry presupposes that exactly one of $\{p, q\}$ is true in the actual world (op. cit. pg. 184), leaving the proposition expressed by the disjunction undefined along the bottom row of the matrix in Figure 4. Lewis's entry (op. cit., pg. 52) would result in a matrix with F-F-F-F along the same row. But-to preview how the semantics will work under deontic operators like ought $(O)$-this will give the wrong intuitive results for the sentences in Ross's Puzzle and FCP. For example, Lewis's entry under $O$ would entail that (4-a) is equivalent to $\ulcorner\hat{O} \perp\urcorner$ in cases where a lazy agent neither (actually) takes out the trash nor (actually) washes the dishes.

It is also worth noting the great deal of post-Groenendijk and Stokhof 1982 work (not in a twodimensional tradition) on the general category of concealed questions into which (6) and (7) falls. For developments in treating this class in inquisitive semantics, see, e.g., Roelofsen 2019. 


\begin{tabular}{c|cc|c|c|}
\multicolumn{1}{c}{} & $w_{1}$ & $w_{2}$ & $w_{3}$ & $w_{4}$ \\
\cline { 4 - 5 }$w_{1}$ & $\mathrm{~T}$ & $\mathrm{~T}$ & $\mathrm{~T}$ & $\mathrm{~F}$ \\
\cline { 5 - 5 }$w_{2}$ & $\mathrm{~T}$ & $\mathrm{~T}$ & $\mathrm{~F}$ & $\mathrm{~F}$ \\
\cline { 3 - 5 }$w_{3}$ & $\mathrm{~T}$ & $\mathrm{~F}$ & $\mathrm{~T}$ & $\mathrm{~F}$ \\
\cline { 3 - 5 }$w_{4}$ & $\mathrm{~T}$ & $\mathrm{~T}$ & $\mathrm{~T}$ & $\mathrm{~F}$ \\
\cline { 2 - 5 } & \multicolumn{4}{c}{$p$ OR $q$}
\end{tabular}

\begin{tabular}{c|c|c|c|c|}
\multicolumn{1}{c}{} & \multicolumn{1}{c}{$w_{1}$} & $w_{2}$ & $w_{3}$ & $w_{4}$ \\
\cline { 2 - 5 }$w_{1}$ & $\mathrm{~T}$ & $\mathrm{~T}$ & $\mathrm{~T}$ & $\mathrm{~F}$ \\
\cline { 2 - 5 }$w_{2}$ & $\mathrm{~T}$ & $\mathrm{~T}$ & $\mathrm{~T}$ & $\mathrm{~F}$ \\
\cline { 2 - 5 }$w_{3}$ & $\mathrm{~T}$ & $\mathrm{~T}$ & $\mathrm{~T}$ & $\mathrm{~F}$ \\
\cline { 2 - 4 }$w_{4}$ & $\mathrm{~T}$ & $\mathrm{~T}$ & $\mathrm{~T}$ & $\mathrm{~F}$ \\
\cline { 2 - 5 } & \multicolumn{4}{c}{$p \vee q$}
\end{tabular}

Figure 4: Matrix for $p$ or $q$, compared with that of $p \vee q$, where $w_{1}$ is an arbitrary $(p \wedge q)$-world, $w_{2}$ is a $(p \wedge \neg q)$-world, $w_{3}$ is a $(\neg p \wedge q)$-world, and $w_{4}$ is a $(\neg p \wedge \neg q)$ world.

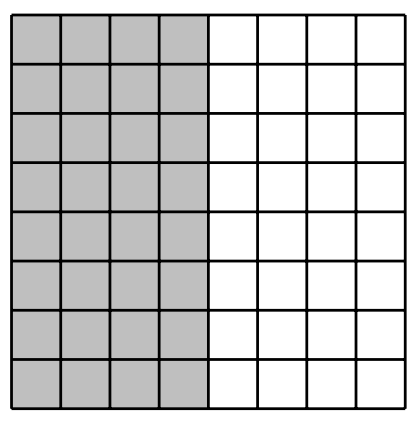

$p$

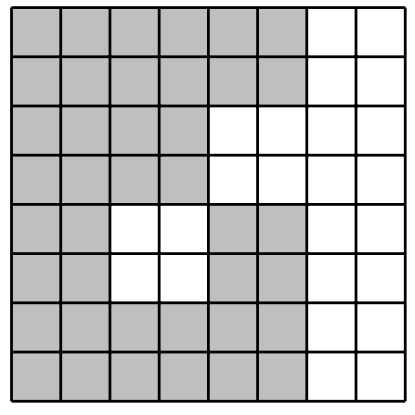

$p$ OR $q$

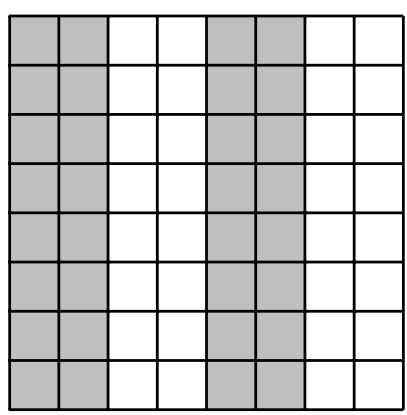

$q$

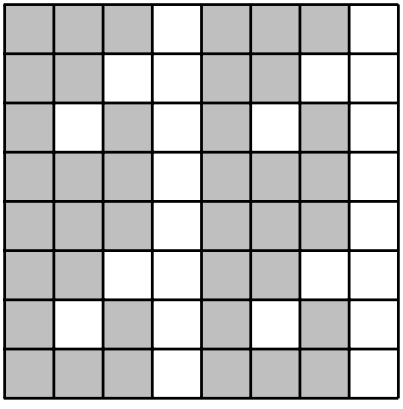

$q$ OR $r$

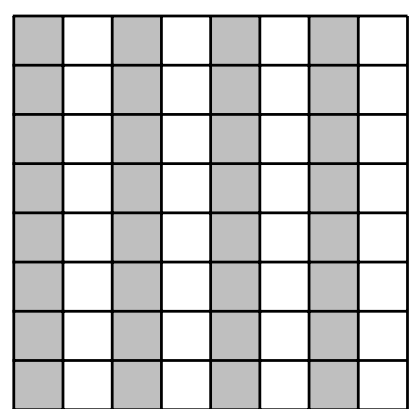

$r$

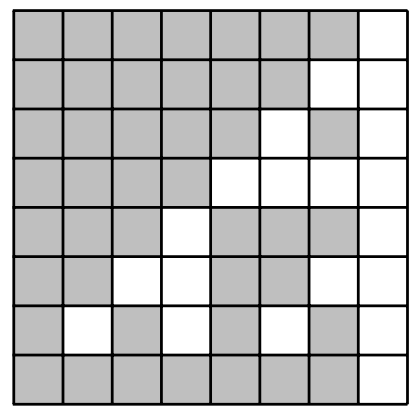

$p$ OR $(q$ OR $r)$

Figure 5: Matrices with 8 worlds per axis. Shading indicates 'true'; white 'false'. 


\subsection{Axiomatics}

What is the logic of two-dimensional or like? While some classical inference patterns governing $\vee$ (e.g., Or-Intro) are not strictly valid for OR, some are. For instance, the following principles are still classically strictly valid even with Fusco's two-dimensional or :

Idempotence. $\phi \models(\phi$ OR $\phi)$

Commutativity. $\phi$ OR $\psi \models \psi$ OR $\phi$

Associativity. $\phi$ OR $(\psi$ OR $\chi) \models(\phi$ OR $\psi)$ OR $\chi^{21}$

Or-Elim. $\phi$ OR $\psi, \neg \phi \models \psi$

Thus, the logic for on is not entirely divorced from the logic of $v$ off the diagonal. But it does raise the question of which principles, exactly, are the result of projecting from the two-dimensional or into a one-dimensional framework. So we turn to the question of how to axiomatize our basic two-dimensional language $\mathcal{L}^{2 \mathrm{D}}$ when extended with or .

In a sense, this task is trivial; as Humberstone (2020) notes, $\phi$ OR $\psi$ on Fusco's semantics can be expressed using @: $:^{22}$

$$
(@(\phi \wedge \neg \psi) \rightarrow \phi) \wedge(@(\neg \phi \wedge \psi) \rightarrow \psi) \wedge(@(\phi \leftrightarrow \psi) \rightarrow(\phi \vee \psi))
$$

Thus, we could simply view or as a defined connective. However, this definition is long-winded; it would be nice to know whether there are more illuminating axioms governing or directly.

Indeed, there are. Let $\mathcal{L}^{2 \mathrm{D}}(\mathrm{oR})$ be the result of extending $\mathcal{L}^{2 \mathrm{D}}$ with a primitive OR. Then, as noted above, we could axiomatize $2 \mathrm{D}$ extended with or using the following defining axiom:

$$
\mathrm{OR}_{d f} \quad(p \text { OR } q) \leftrightarrow[(@(p \wedge \neg q) \rightarrow p) \wedge(@(\neg p \wedge q) \rightarrow q) \wedge(@(p \leftrightarrow q) \rightarrow(p \vee q))]
$$

Alternatively, we could replace or $d f$ with the following six axioms, which more closely mirror the standard introduction and elimination rules for disjunction:

$$
\begin{array}{ll}
\text { OR } I_{1} & (@ p \wedge p) \rightarrow(p \text { oR } q) \\
\text { OR } I_{2} & (@ q \wedge q) \rightarrow(p \text { OR } q) \\
\text { OR } I_{3} & (@ \neg(p \vee q) \wedge(p \vee q)) \rightarrow(p \text { OR } q)
\end{array}
$$

\footnotetext{
${ }^{21}$ The validity of Associativity can be verified by cases based on which of $\phi, \psi$, and $\chi$ is satisfied at $\langle y, y\rangle$. We omit the proof, as it is tedious and unilluminating. Note also that the converse of Associativity follows from Associativity and Commutativity.

${ }^{22}$ In fact, the opposite is true, too: @ $\phi$ can be expressed as $(\phi$ OR $\neg \phi) \leftrightarrow \phi$.
} 
OR $E_{1} \quad(p$ OR $q) \rightarrow(p \vee q)$

OR $E_{2} \quad((p$ OR $q) \wedge @ \neg p) \rightarrow(@ \neg q \vee q)$

OR $E_{3} \quad((p$ oR $q) \wedge @ \neg q) \rightarrow(@ \neg p \vee p)$

It is straightforward to show that $\mathbf{2} \mathbf{D}+$ OR $d f$ is equivalent to $\mathbf{2} \mathbf{D}+$ OR $I_{1}-$ OR $E_{3}$. The latter, however, more clearly manifest the way in which the rules governing $\vee$ can be seen as one-dimensional projections from more general two-dimensional rules governing OR. Since @ $\phi$ is diagonally equivalent to $\phi$, OR $I_{1}$ and OR $I_{2}$ reduce to the standard disjunction introduction rule; or $I_{3}$ diagonally follows from explosion; oR $E_{1}$ is already an articulation of the disjunction elimination rule in terms of disjunctive syllogism; and $\mathrm{OR} E_{2}$ and $\mathrm{OR} E_{3}$ diagonally follow from the fact that everything implies a tautology.

Using these axioms, we can directly derive the collapse of or and $\vee$ along the diagonal. To facilitate the reasoning, observe that the following rule is admissible in $2 \mathrm{D}$ for any primitive modal operator $\triangle$ :

RK. If $\phi_{1}, \ldots, \phi_{n} \models \psi$, then $\triangle \phi_{1}, \ldots, \triangle \phi_{n} \models \Delta \phi$.

Here, then, is an axiomatic proof of $\square \dagger((\phi$ oR $\psi) \leftrightarrow(\phi \vee \psi))$ - the a priori equivalence of OR and $\vee$ (with instances of Uniform Substitution suppressed):

1. $(\phi$ OR $\psi) \rightarrow(\phi \vee \psi)$

OR $E_{1}$

2. $\dagger((\phi$ OR $\psi) \rightarrow(\phi \vee \psi))$

Necessitation, 1

3. $\dagger(\phi \rightarrow @ \phi)$ $X \rightarrow$

4. $\dagger(\psi \rightarrow @ \psi)$ $X \rightarrow$

5. $(@ \phi \wedge \phi) \rightarrow(\phi$ OR $\psi)$ OR $I_{1}$

6. $(@ \psi \wedge \psi) \rightarrow(\phi$ OR $\psi)$ OR $I_{2}$

7. $\dagger((@ \phi \wedge \phi) \rightarrow(\phi$ OR $\psi))$ Necessitation, 5

8. $\dagger((@ \psi \wedge \psi) \rightarrow(\phi$ OR $\psi))$ Necessitation, 6

9. $\dagger(\phi \rightarrow(\phi$ OR $\psi))$ RK, 3, 7

10. $\dagger(\psi \rightarrow(\phi$ OR $\psi))$ RK, 4, 8

11. $\dagger((\phi$ OR $\psi) \leftrightarrow(\phi \vee \psi))$ RK, 2, 9, 10

12. $\square \dagger((\phi$ OR $\psi) \leftrightarrow(\phi \vee \psi))$ Necessitation, 11

This offers an axiomatic illustration of how the two-dimensional entry for or is an isotope of the standard boolean $v$. 


\section{Deontic Modality}

\subsection{Two-Dimensional Deontic Logic}

Here is an interim summary of where we are. In $\S \S 2.1-2.2$, we presented several motivations for the two-dimensional framework as well as four notions of consequence that can be distinguished within it. The axiomatization of this framework was given in $\S 2.3$. In $\S 2$.4, we motivated exploring Åqvist logics with their $\AA$ compliant atomics. Finally, in $\S 3$, we sketched a two-dimensional semantics of disjunction and presented an axiomatization for it.

With this setup, we are ready to enrich the language with the deontic operators $O$ and $M$. We do this by enriching the modal domain of our matrices with a deontic accessibility relation $R_{O}$. The goal of this section is to articulate the logic that results from lifting standard one-dimensional deontic logic into two-dimensions. We then show how doing so can solve the deontic puzzles with which we began.

First, however, we face a choice point regarding the deontic accessibility relation, similar to the choice point faced by the fully general vs. Åqvist approach to atomics sketched in $\S 2.4$, viz., should the deontic accessibility relation be uniform across each row of the matrix? A 'yes' answer suggests that at its foundations, deontic accessibility cannot depend on facts which are (metaphysically or epistemically) indeterminate relative to the context set. A 'no' answer, on the other hand, suggests that it can.

Here, we present completeness results for both paths from this choice point. However, a number of interpretative complications arise on the latter, non-uniform approach. (What does it mean, exactly, for deontic ideality to depend both on the world-as-actual and the world of evaluation?) And, at any rate, Fusco (2015) adopts a uniform deontic accessibility relation. Since our main goal is to axiomatize this system, we will allow uniformity to play a role in explaining Ross's Puzzle and (more critically) FCP. We leave it to future investigation to settle whether the uniformity approach is warranted.

Second, there is another choice point concerning the properties of the deontic accessibility relation. In standard one-dimensional deontic logic, it is assumed that the deontic accessibility relation is at the very least a serial and shift-reflexive subrelation of $R_{\square}$ (generalized to the two-dimensional setting; for readability, we leave off the world-as-actual parameter, which is held constant throughout).

May Implies Can. $\forall w, v: w R_{O} v \Rightarrow w R_{\square} v$

Seriality. $\forall w \exists v: w R_{O} v$

Shift-Reflexivity. $\forall w, v: w R_{O} v \Rightarrow v R_{O} v$ 
These constraints correspond respectively to the following axioms (where $\hat{O}$ is defined to be the dual of $O$; we separate $\hat{O}$ from $M$ conceptually for reasons that will be made clear in $\S 4.3$ ):
$M C \quad \hat{O} p \rightarrow \diamond p$
$D_{O} \quad \mathrm{Op} \rightarrow \hat{\mathrm{O} p}$
$O T_{O} \quad O(O p \rightarrow p)$

Adding $D_{O}$ and $O T_{O}$ to the minimal normal modal logic results in the modal logic KDU, i.e., "standard deontic logict" in the terminology of McNamara 2010.

Stronger constraints could be imposed on $R_{O}$, though. In particular, it is natural to assume that $R_{O}$ is rigid, meaning which worlds are "deontically ideal" does not vary from world to world:

Deontic Rigidity. $\forall w, v, u: w R_{\square} v \& w R_{O} u \Rightarrow v R_{O} u$

This corresponds to the following axiom:

$D R \quad \hat{O} p \rightarrow \square \hat{O} p$

On this analysis, $R_{O}$ effectively partitions $W$ into the classes of deontically ideal and nonideal worlds. ${ }^{23}$ This allows us to reduce deontic logic to alethic logic via the standard Anderson-Kanger reduction using $\square$ and a privileged atomic $d$ standing for $\ulcorner$ The deontic ideals are met $\urcorner$ (Anderson, 1958; Kanger, 1971):

$$
\begin{aligned}
O \phi & :=\square(d \rightarrow \phi) \\
M \phi & :=\diamond(d \wedge \phi)
\end{aligned}
$$

Again, for the sake of neutrality, we present completeness results for both options. It turns out that this option affects what we say about FCP. Because Fusco only adopts the weaker framework, without Deontic Rigidity, she needs to revise the relationship between $O$ and $M$ in order to explain FCP. Assuming Deontic Rigidity, however, this revision is not required.

Let us now turn to the question of how to axiomatize each combination of choices.

Definition 6. The two-dimensional deontic language $\mathcal{L}^{\mathrm{D} 2 \mathrm{D}}$ is defined recursively as follows:

$$
\phi::=\operatorname{Prop}|\neg \phi|(\phi \wedge \phi)|\square \phi| @ \phi|\dagger \phi| O \phi .
$$

In addition to the other abbreviations, we define $\hat{O} \phi:=\neg O \neg \phi$.

\footnotetext{
${ }^{23}$ This is similar to the way the deontic selection function of MacFarlane and Kolodny (2010) works. See also Kratzer 1977, 1981's ordering-source approach.
} 
Definition 7. A deontic matrix frame is a tuple $\mathcal{F}=\left\langle W \times W, R_{\square}, R_{\Theta}, R_{\dagger}, R_{O}\right\rangle$, where $\left\langle W, R_{\square}, R_{\Theta}, R_{\dagger}\right\rangle$ is a basic matrix frame and $R_{O}$ is a serial, shift-reflexive subrelation of $R_{\square}$. A deontic matrix model is a pair of a deontic matrix frame with a valuation function $V$ : Prop $\rightarrow \wp(W \times W)$. A deontic matrix frame (model) is rigid if in addition $R_{O}$ satisfies Deontic Rigidity. Let DM be the class of deontic matrix models and DMR the class of rigid deontic matrix models.

Definition 8. Let D2D be the logic that results from adding $M C-O T_{O}$ as axioms to 2D, and let D2DR be the result of adding $D R$ as well.

The following is proved in $\S \mathrm{A}$.

\section{Theorem 3.}

(a) D2D is sound and complete for DM.

(b) D2DR is sound and complete for DMR.

Now for adding the other options.

Definition 9. Let $\mathcal{M}=\left\langle W \times W, R_{\square}, R_{\circledast}, R_{\dagger}, R_{O}, V\right\rangle$ be a deontic matrix model. Say $R_{O}$ is uniform if the following condition is met for all $x, y, w, v \in W$ :

$$
\langle\langle x, w\rangle,\langle x, v\rangle\rangle \in R_{O} \quad \Leftrightarrow \quad\langle\langle y, w\rangle,\langle y, v\rangle\rangle \in R_{O} .
$$

We'll say $\mathcal{M}$ is uniform if $R_{O}$ is. The definition of Aqvist matrix models from Definition 4 carries over to deontic matrix models. Let DMÅU be the class of Åqvist uniform deontic matrix models and DMÅUR the class of Åqvist, uniform, and rigid deontic matrix models.

To state the proof system, we first need a definition.

Definition 10. A $\mathcal{L}^{\mathrm{D} 2 \mathrm{D}}$-formula is explicitly $1 \mathrm{D}$ if it is $@$-free and $\dagger$-free.

Definition 11. Let $\mathbf{F}:=$ D2DÅU be the proof system axiomatized by Modus Ponens, Necessitation for each primitive modal operator, $\AA$, and the following axiom schemas:

$D 2 D \phi$, where $\phi$ is a theorem of D2D.

$U \quad O \phi \leftrightarrow \dagger O \phi$ where $\phi$ is an explicitly 1D-formula.

Let FR := D2DÅUR be defined similarly except we replace $D 2 D$ with:

D2DR $\phi$, where $\phi$ is a theorem of D2DR. 
F is the proof system that axiomatizes Fusco (2015)'s semantics, while FR is the same proof system extended with $D R$. The following is proved in $\S B .{ }^{24}$

\section{Theorem 4.}

(a) F is sound and complete for DMÅU.

(b) FR is sound and complete for DMÅUR.

F and FR give us a pair of one-dimensional deontic logics "lifted" into two dimensions - that is, into the space of matrices-in a way that preserves the original, one-dimensional interpretation of atoms. ${ }^{25}$

\subsection{Ross in the context of Deontic Matrix Models}

Now that we've seen various ways of lifting deontic logic into two dimensions, what happens when we adopt Fusco's two-dimensional semantics for or? As it turns out, both Ross's Puzzle and FCP can be accounted for (though, as we'll see, the latter may require a bit extra work to obtain).

Start with Ross's Puzzle. Even in FR, the Ross inference is not a priori valid: $O p \triangleright_{\text {DMÅUR }} O(p$ OR $q)$. A counterexample is given in Figure 6. Let us say a model $\mathcal{M}$ a priori satisfies $\phi$, written $\mathcal{M} \triangleright \phi$, if $\mathcal{M}, z, z \Vdash \phi$ for all $z \in W$. (Thus, $\Gamma \triangleright_{\mathrm{C}} \phi$ iff for every $\mathcal{M} \in \mathrm{C}$, if $\mathcal{M} \triangleright \Gamma$, then $\mathcal{M} \triangleright \phi$.) The deontic matrix model in Figure 6 a priori satisfies the premise $O p$. But it fails to a priori satisfy the conclusion $O(p$ OR $q)$. The witness for this failure is $\left\langle w_{q}, w_{q}\right\rangle$ : at $\left\langle w_{q}, w_{q}\right\rangle$, the disjunction $p$ or $q$ expresses the proposition that $q$. But $q$ is not obligatory (or even permissible) at $\left\langle w_{q}, w_{q}\right\rangle$.

To get a better sense of why failures of the Ross inference arise, it helps to contrast this counterexample with a model where $O(p$ OR $q)$ is a priori satisfied, such as in Figure 7. We can see that $q$-the actually true answer to $\ulcorner p$, or $q$ ? $\urcorner$ in $w_{q}$-is obligatory from the point of view of $\left\langle w_{q}, w_{q}\right\rangle .{ }^{26}$ We can also see that $p$ - the actually true answer to $\ulcorner p$, or $q$ ? $\urcorner$ in $w_{p}$-is obligatory from the point of view of $\left\langle w_{p}, w_{p}\right\rangle$, the other diagonal, or 'a priori', point in the model. If it is $q$ which the agent actually does-settling that she is at the diagonal point on the second row of the matrix-then it is $q$ she ought to do. But the reverse is true if it is $p$ which she actually does. Since the choice of actuality is up to her, what is deontically

\footnotetext{
${ }^{24} \mathrm{An}$ anonymous reviewer asks whether consequence over DMU can be axiomatized by just adding $U$ to D2D. Alternatively, one might conjecture that we can drop $\AA$ from $\mathbf{F}$. The answer is negative on both counts, since $U$ is not sound for the class of (even rigid) uniform deontic matrix models that are not Åqvist. A counterexample is given in $\S$ B (Figure 11).

${ }^{25}$ These logics hence preserve the one-dimensional notion of "good arguments" from Gregory 2001 and Williamson 2013 (glossed in footnote 19).

${ }^{26}$ To simplify the visuals, $p$ and $q$ are represented in Figures $6-7$ as mutually exclusive and jointly exhaustive.
} 


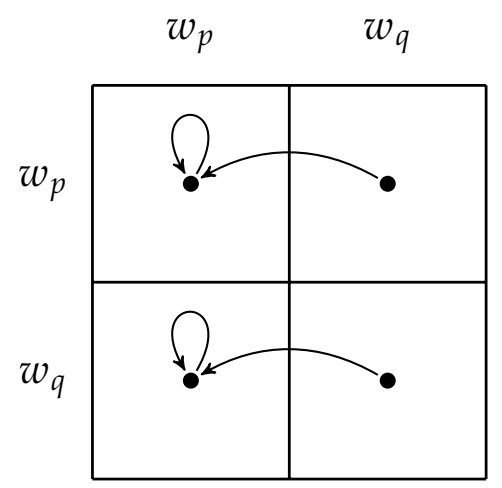

Figure 6: A counterexample to Ross's Puzzle in FR, where for any $\alpha, \beta, \gamma \in$ Prop, $\left\langle w_{\beta}, w_{\gamma}\right\rangle \in V(\alpha)$ iff $\gamma=\alpha$.

ideal is also up to her: she has a deontically free choice in Figure 7 which she lacks in Figure 6. ${ }^{27}$

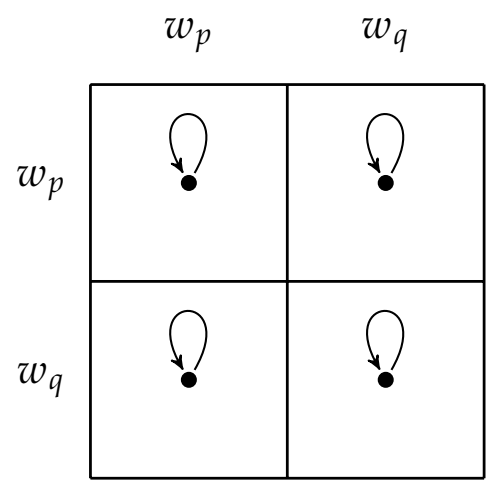

Figure 7: A model where $O(p$ or $q)$ is a priori satisfied.

\subsection{Permissibility and Free Choice Permission}

Let's turn now to FCP. Before we explain how two-dimensional deontic logic captures this principle, we add a qualification. The FCP inference seems to be licensed for both disjuncts only when it's possible for the agent to make each disjunct true without the other. For instance, suppose you are told you may take

\footnotetext{
${ }^{27}$ It is worth noting that a proposal for understanding FCP which is similar in spirit but quite different in implementation to the present approach, is independently developed in the linguistics literature by Kaufmann (2016). Unfortunately, we lack the space to compare the similarities and differences of the views at length here.
} 
an apple or a pear, but it turns out (say, for practical reasons) that you can only take the pear if you also take the apple. In that case, it seems as though you are not, in general, permitted to take the pear. In light of this, the form of free choice we will be interested in makes explicit that it's possible for each disjunct to be true without the other: ${ }^{28}$

FCP $^{*} . M(\phi$ OR $\psi), \diamond(\phi \wedge \neg \psi), \diamond(\psi \wedge \neg \phi) \models M \phi \wedge M \psi$

We'll start with deontically rigid models, since the explanation of $\mathrm{FCP}^{*}$ over this class is simpler. Three ingredients are required. First, $M$ is the dual of $O$, i.e., $M:=$ Ô. Second, the deontic accessibility relation is uniform. And third, the formulas involved in the inference are Aqvist, i.e., their propositional content does not vary with the world-as-actual. Thus, where the $\AA$ operator is defined as in $\S 2.4$, we have the following free choice theorem: ${ }^{29,30}$

Theorem 5. $M(\phi$ OR $\psi), \diamond(\phi \wedge \neg \psi), \AA \phi, \AA \psi \triangleright_{\text {DMÅUR }} M \phi$.

Proof. Let $\mathcal{M}$ be a Åqvist, uniform, and rigid deontic matrix model such that:

(i) $\mathcal{M} \triangleright M(\phi$ or $\psi)$

(ii) $\mathcal{M} \triangleright \diamond(\phi \wedge \neg \psi)$

(iii) $\mathcal{M} \triangleright \AA \phi \wedge \AA \psi$

Let $w \in W$; we will show that $\mathcal{M}, w, w \Vdash M \phi$. By (ii), there exists a $v \in W$ such that $\mathcal{M}, w, v \Vdash \phi \wedge \neg \psi$. By (iii), $\mathcal{M}, w, v \Vdash \phi \wedge \neg \psi$ iff $\mathcal{M}, w, v \Vdash \dagger \phi \wedge \neg \dagger \psi$, which holds iff $\mathcal{M}, v, v \Vdash \phi \wedge \neg \psi$. Hence, $\operatorname{Ans}(v, \phi, \psi)=\{\phi\}$. By (i), $\mathcal{M}, v, v \Vdash$ $M(\phi$ or $\psi)$. So there exists a $u \in W$ such that $\langle v, v\rangle R_{O}\langle v, u\rangle$ and $\mathcal{M}, v, u \Vdash \phi$ or $\psi$. Since $\operatorname{Ans}(v, \phi, \psi)=\{\phi\}$, that means $\mathcal{M}, v, u \Vdash \phi$. By (iii), $\mathcal{M}, w, u \Vdash \phi$. But because $R_{O}$ is uniform, $\langle w, v\rangle R_{O}\langle w, u\rangle$. And because $R_{O}$ is rigid, that means $\langle w, w\rangle R_{O}\langle w, u\rangle$. Hence, $\mathcal{M}, w, w \Vdash M \phi$.

${ }^{28}$ Empirical data suggests these this caveat is connected to the exclusivity data from footnote 1 , especially in the case where there are more than two disjuncts. In the simplest case, suppose that for the disjunction $\left\ulcorner p_{1}\right.$ OR $p_{2}$ OR $\ldots$ OR $\left.p_{n}\right\urcorner$ it holds that $\square\left(p_{i} \supset p_{i+1}\right)$. Then it is impossible to make $p_{1}$ true without making $\wedge_{i} p_{i}$ true. In this case, FCP, but not FCP, would hold that $M\left(p_{1}\right.$ OR $\ldots$ OR $\left.p_{n}\right)$ entails $M\left(\bigwedge_{i} p_{i}\right)$, contrary to EX from footnote 1 . Recent experimental work suggests this entailment is not licensed in these cases (Fusco, 2020a).

${ }^{29}$ In fact, Theorem 5 holds even for the class of uniform and rigid deontic matrix models-that is, we don't have to require valuations to be Åqvist. But the completeness proof of $\mathbf{F}(\mathbf{R})$ requires the deontic matrix models be Åqvist, so we've stated the theorem in terms of Åqvist models.

${ }^{30} \mathrm{An}$ anonymous reviewer points out that $\triangle_{\mathrm{DM}} \mathrm{A} \cup \mathrm{R}$ may be too strong to plausibly formalize natural language entailment. For instance, $p \triangleright_{\mathrm{DMA} U \mathrm{U}} O p$ and $p \triangleright_{\mathrm{DMA} U \mathrm{R}} \square p$. This is because the atoms are Åqvist, so if they're true at every diagonal, they're true everywhere. (More generally, $\phi, \AA \phi \triangleright_{\mathrm{DM}} \square \phi$ and $\left.\phi, \AA \phi \triangleright_{\mathrm{DM}} O \phi.\right)$ This is related to the "If $p$, Ought $p$ " problem, which information-sensitive theories of 'ought' tend to face. See Carr 2014 for discussion. We do not propose a solution to this problem, though we suspect it will require a better understanding of how conditionals relate to disjunction (observe, e.g., that $\searrow_{\mathrm{DMA} U \mathrm{R}} \neg p$ OR $O p$ ). 
We can illustrate this using by contrasting Figure 8 and Figure 6. In Figure 8, $M(p$ OR $q)$ is a priori satisfied because no matter which world is actual, the actual answer to $\ulcorner p$, or $q$ ? $\urcorner$ is permissible. By contrast, in Figure $6, q$ is not permissible, and so $M(p$ or $q)$ is not a priori satisfied—specifically, it's not satisfied at $\left\langle w_{q}, w_{q}\right\rangle$.

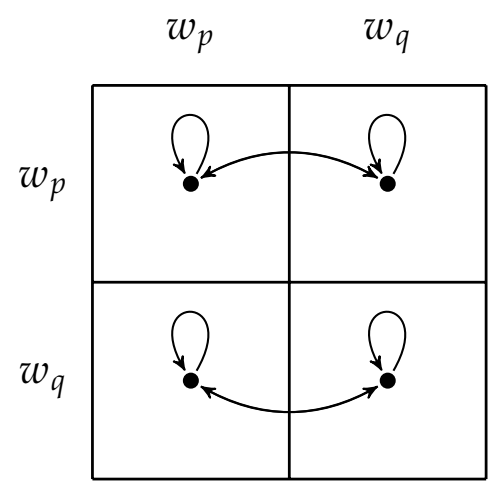

Figure 8: $\mathcal{M} \triangleright M(p$ or $q)$

The addition of premises $\AA \phi$ and $\AA \psi$ is new relative to Fusco 2015. There, Fusco states the free choice inference (using our notation) as:

$$
M(\phi \text { OR } \psi), \diamond(\phi \wedge \neg \psi), \diamond(\neg \phi \wedge \psi) \triangleright M \phi \wedge M \psi
$$

where $\phi$ and $\psi$ are both disjunction-free and non-modal. In that framework, Fusco implicitly assumes that all atomics are Åqvist, which entails that any such formulas are Åqvist. Thus, our theorem is a generalization of Fusco's result: her free choice inference can be extended to any $\phi$ and $\psi$ so long as their content does not vary from row-to-row.

There are cases involving non-Åqvist disjuncts, however, where free choice does not hold in this semantics. Here is a counterexample. Our matrix model $\mathcal{M}$ will consist of four worlds $w_{p q}, w_{\bar{p} q}, w_{p \bar{q}}, w_{\bar{p} \bar{q}}$. Our (Åqvist) valuation will be the obvious one:

$$
\begin{aligned}
& \left\langle w_{\alpha \beta}, w_{\gamma \delta}\right\rangle \in V(p) \Leftrightarrow \gamma=p \\
& \left\langle w_{\alpha \beta}, w_{\gamma \delta}\right\rangle \in V(q) \Leftrightarrow \delta=q .
\end{aligned}
$$

The deontic accessibility relation will say all and only $\bar{p}$-worlds are deontically ideal:

$$
\left\langle w_{\alpha \beta}, w_{\gamma \delta}\right\rangle R_{O}\left\langle w_{\alpha \beta}, w_{\gamma^{\prime} \delta^{\prime}}\right\rangle \quad \Leftrightarrow \quad \gamma^{\prime}=\bar{p}
$$

Note, $R_{O}$ is both uniform and rigid. This model is summarized in Figure 9 . 

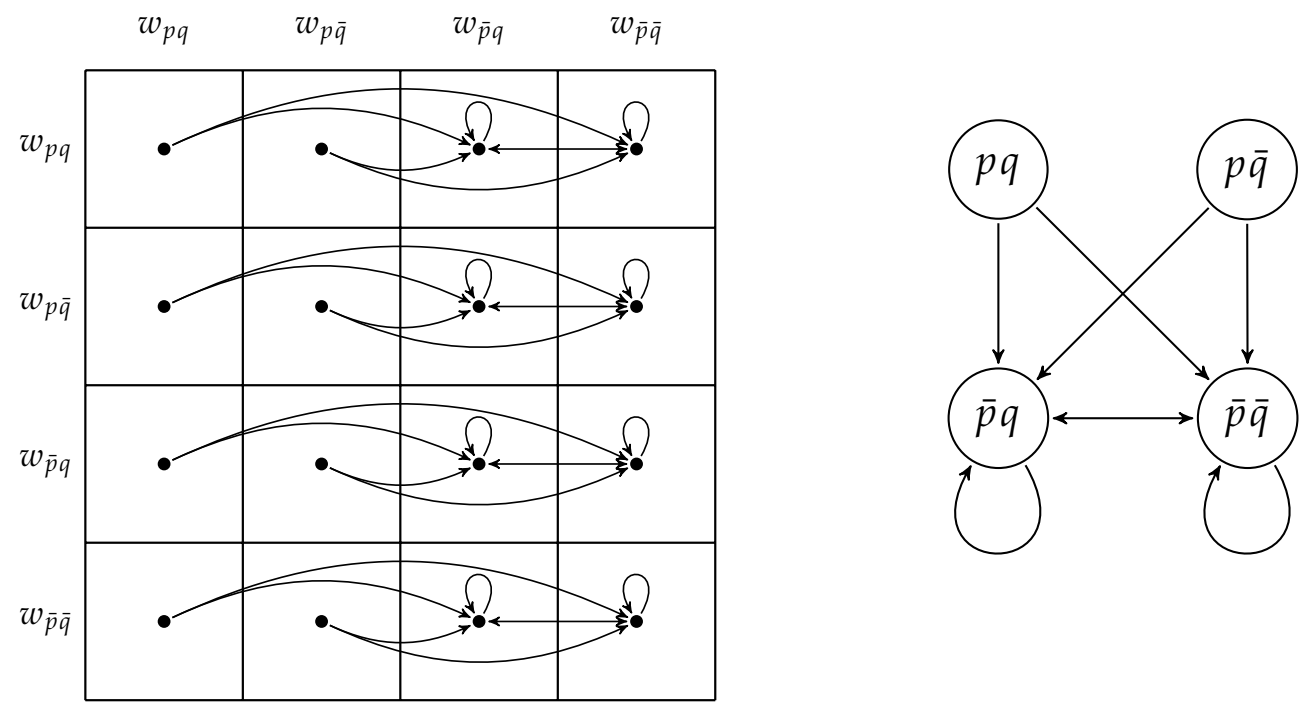

Figure 9: Counterexample to free choice with non-Åqvist disjuncts. The diagram on the right is a visualization of the accessibility relations on each row.

Then letting $\phi=@ p \leftrightarrow p$ and $\psi=@ q \leftrightarrow q$, we have: ${ }^{31}$

$$
\begin{aligned}
& \mathcal{M} \triangleright M((@ p \leftrightarrow p) \text { OR }(@ q \leftrightarrow q)) \\
& \mathcal{M} \triangleright \diamond((@ p \leftrightarrow p) \wedge \neg(@ q \leftrightarrow q)) \\
& \mathcal{M} \triangleright \diamond(\neg(@ p \leftrightarrow p) \wedge(@ q \leftrightarrow q)) \\
& \mathcal{M} \triangleright M(@ p \leftrightarrow p)
\end{aligned}
$$

Thus, FCP* does not hold for all non-Åqvist cases in Fusco's semantics.

With that said, we think this is not a serious cost to Fusco's approach, for two reasons. First, these sorts of counterexamples are quite strange. It is hard to see how we could have robust and reliable intuitions about their analogues in natural language. This is especially complicated by evidence that the actually operator @ is not be an adequate formalization of the English word 'actually'. ${ }^{2}$ So these consequences of Fusco's semantics would likely be hard to test empirically.

Second, there is an alternative formulation of free choice available that avoids

\footnotetext{
${ }^{31}$ To see why, note that for each $v \in W, A n s(v, @ p \leftrightarrow p, @ q \leftrightarrow q)=\{@ p \leftrightarrow p, @ q \leftrightarrow q\}$. This is because $@ \phi \leftrightarrow \phi$ is a diagonal validity. Thus, for each $v \in W$, we have $\mathcal{M}, v, v \Vdash M((@ p \leftrightarrow p)$ oR $(@ q \leftrightarrow q))$ iff $\exists \delta \in\{q, \bar{q}\}: \mathcal{M}, v, w_{\bar{p} \delta} \Vdash(@ p \leftrightarrow p) \vee(@ q \leftrightarrow q)$. It is easily verified this holds for all $v$, since some deontically ideal world agrees with $v$ on $q$. Moreover, the possibility premises are satisfied on every row, since every combination of truth values to $p$ and $q$ is realized. But while $\mathcal{M} \triangleright M(@ q \leftrightarrow q)$, we do not have $M(@ p \leftrightarrow p)$ satisfied at every diagonal point. Specifically, $\mathcal{M}, w_{p q}, w_{p q} \forall / M(@ p \leftrightarrow p)$ and $\mathcal{M}, w_{p \bar{q}}, w_{p \bar{q}} \not \nvdash M(@ p \leftrightarrow p)$.

${ }^{32}$ For data showing that English 'actually' is more complex, see Yalcin (2015).
} 
these complications. We can drop the premises $\AA \phi$ and $\AA \psi$ from the inference so long as we diagonalize the disjuncts first.

Theorem 6. $M(\dagger \phi$ OR $\dagger \psi), \diamond(\dagger \phi \wedge \neg \dagger \psi) \triangleright_{\mathrm{DMA} U \mathrm{UR}} M \dagger \phi$

Proof. Immediate from Theorem 5 and the fact that $\models_{\mathrm{DM}} \AA \dagger \phi$.

In fact, Theorem 5 can be seen as a special instance of this more general principle, since $\AA \phi \models \square(\phi \leftrightarrow \dagger \phi)$. We often diagonalize material in the scope of modals as part of a broader reinterpretation strategy. For instance, while (8) is not metaphysically necessary, and thus (9) is false so-interpreted, there does seem to be a reading of (9) on which it is true, viz., one where the flavor of necessity is a priority.

(8) I am here now.

(9) Necessarily, I am here now.

a. False: $\square(\mathrm{I}$ am here now $)$

b. True: $\square \dagger($ I am here now $)$

It would therefore be unsurprising if diagonalization occurred in the scope of disjunction, given that disjunction is itself interpreted as a kind of binary modal.

This concludes the explanation of free choice when the accessibility relation is deontically rigid. Let us now turn to the case where it is not. In fact, Fusco (2015) does not endorse deontic rigidity because of cases like the following:

Choosing Childbearing. You face a choice between conceiving a child early in your life $[=p]$ or a different child significantly later $[=q]$. You believe that your values will be transformed by the choice you make. In particular, because the choice you make will be a necessary condition for the existence of a person you will love, you will affirm that choice over any other. There is no single psychological standpoint that values both of these potential persons to equal degree. (Parfit, 1984, pg. 360-361; Paul, 2014, Ch. 3; Paul, 2015.)

Nice Choices at the Spa. Aromatherapy $[=p]$ or body-wrap $[=q]-$ which is it to be? You believe that, whichever you choose, you will be very glad you chose it. Mid-aromatherapy, the aromatherapy will seem self-evidently superior [to the body-wrap]. Mid-body-wrap, the bodywrap will seem self-evidently superior [to the aromatherapy]. (Hare and Hedden, 2016, pg. 3)

Suppose "nice choices" like the ones described above are possible. Then worlds where the agent makes different choices may have different deontic points of view. This would require abandoning Deontic Rigidity as a constraint on the deontic accessibility relation. 


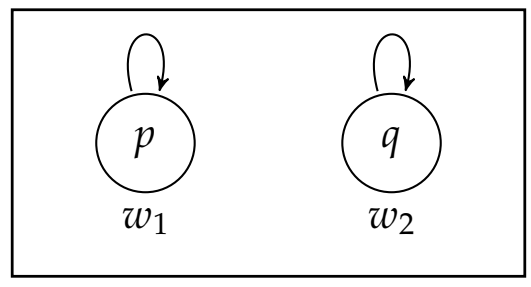

Figure 10: A one-dimensional nice choice

To accommodate free choice inferences in this more general framework, Fusco proposes to capture $\mathrm{FCP}^{*}$ by reconceiving of the connection between obligation and permission. In brief, where the standard view is that $M$ is just $\hat{O}$, i.e., the dual of $O$, Fusco proposes to analyze $M$ in terms of a weaker condition, viz., $\diamond \hat{O}$. Thus, the revised truth conditions for $M$ are as follows:

$\left(M_{2}\right) \quad y, x \Vdash M \phi$ iff there is some $x^{\prime}$ such that $x^{\prime} R_{O} x^{\prime}$ and $y, x^{\prime} \Vdash \phi$

Redefining $M$ in this way, we obtain free choice once again:

Theorem 7. Where $M:=\diamond \hat{O}$ :

(a) $M(\phi$ OR $\psi), \diamond(\phi \wedge \neg \psi), \AA \phi, \AA \psi \triangleright_{\mathrm{DM}} \mathrm{M} \mathrm{M} \phi$

(b) $M(\dagger \phi$ OR $\dagger \psi), \diamond(\dagger \phi \wedge \neg \dagger \psi) \triangleright_{\mathrm{DMÅU}} M \dagger \phi$

Proof. The proof of (a) is the same as that of Theorem 5 up to where we inferred that $\mathcal{M}, v, v \Vdash M(\phi$ or $\psi)$. From there, it follows that there exists a $u \in W$ such that for some $u^{\prime} \in W,\langle v, u\rangle R_{O}\left\langle v, u^{\prime}\right\rangle$ and $\mathcal{M}, v, u^{\prime} \Vdash \phi$ OR $\psi$. Since $\operatorname{Ans}(v, \phi, \psi)=\{\phi\}$, that means $\mathcal{M}, v, u^{\prime} \Vdash \phi$. By (iii), $\mathcal{M}, w, u^{\prime} \Vdash \phi$. But because $R_{O}$ is uniform, $\langle w, u\rangle R_{O}\left\langle w, u^{\prime}\right\rangle$. Hence, $\mathcal{M}, w, u \Vdash \hat{O} \phi$. So $\mathcal{M}, w, w \Vdash \diamond \hat{O} \phi=M \phi$. The proof of (b) is immediate as before.

Thus, one does not need to commit oneself to the claim that each world must agree with every other about which worlds are deontically ideal in order to capture free choice, so long as we take a revisionary stance on the relationship between 'may' and 'ought'.

Note that, in deontically rigid models, this definition of $M$ is equivalent to $O$ :

Theorem 8. $\models_{\mathrm{DMR}} \hat{O} \phi \leftrightarrow \diamond \hat{O} \phi$.

Thus, one could in principle adopt this definition $(M:=\diamond \hat{O})$ in FR as well; it's just that doing so would not be a genuine departure from the orthodox view that 'may' is the dual of 'ought'. 


\section{Conclusion}

In this paper, we explored several ways of axiomatizing $\square, \dagger, @$, and the deontic operators $O$ and $M$, with an eye to FCP and Ross's Puzzle, following the path sketched by Fusco $(2015,2019)$. The language also provides a way of connecting the a priori $(A)$ operator to Stalnaker's "Assertion", via the idea that a natural notion of global or informational consequence is in fact a diagonalized one as well. We axiomatized Fusco's logic of disjunction using rules that collapse into the standard disjunction introduction/elimination rules in the one-dimensional setting. We then explored several different formulations of two-dimensional deontic logic and how these different choice points relate to the deontic puzzles in $\S 1$.

While our focus has primarily been on deontic logic, the various choice points in $\S 4$ highlight a broader lesson for the study of two-dimensional logics. When lifting a one-dimensional system into two dimensions, one needs to keep in perspective the original motivations for doing so. For some applications, it will be desirable not to impose uniformity on accessibility relations, thus preserving the full generality of the two-dimensional framework. But for other applications to natural language, especially in the context of a Stalnakerian picture of assertion, uniformity may be motivated by more than mere convenience. We leave further discussion of these issues for another time.

\section{A Axiomatizing D2D}

In this appendix, we prove Theorem 3: $\mathbf{D 2 D ( R )}$ is sound and complete for the class DM(R) of (rigid) deontic matrix models. The proof is an extension of the completeness results in Fritz 2014; Fusco 2020b for two-dimensional languages without deontic operators. Below, we have omitted the proofs of all the "Facts", as they can be found in Fusco 2020b (or are straightforward extensions of facts therein).

The proof strategy is in three steps. We identify three classes of frames:

1. $\operatorname{Fr}_{\mathbf{D} 2 \mathrm{D}(\mathrm{R})}$, the class of D2D(R)-frames;

2. $R_{D(R)}$, an intermediate class of (rigid) deontic Restall frames;

3. $M F_{D(R)}$, the class of (rigid) deontic matrix frames.

The first step is to establish the soundness and completeness of D2D(R) over

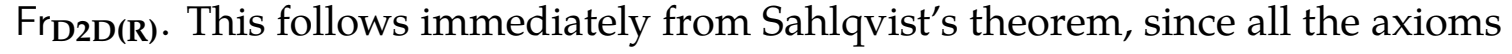
are Sahlqvist formulas. ${ }^{33}$

\footnotetext{
${ }^{33}$ In fact, as an anonymous reviewer notes, this is the only place where we appeal to $D_{O}$ and $O T_{O}$. Thus, the proof strategy generalizes to any set of axioms governing $O$ that are canonical in
} 
Theorem 9. D2D(R) is sound and strongly complete with respect to $\operatorname{Fr}_{\mathbf{D} 2 \mathbf{D}(\mathbf{R}) \text {. }}$

Proof. By Sahlqvist's Theorem (Blackburn et al., 2002, Ch. 4).

The second step is to show that $\operatorname{Fr}_{\mathbf{D} 2 \mathrm{D}(\mathrm{R})}$ is modally equivalent to an intermediate class of frames $R_{D(R)}$, viz., (rigid) deontic Restall frames. This is established by showing that $R_{D(R)}$ is the class of point-generated subframes of $\operatorname{Fr}_{\mathbf{D} 2 \mathrm{D}(\mathrm{R}) \text {. The }}$ final step is then to show that every deontic Restall frame is equivalent to a matrix frame by showing how to construct a bounded morphism from an arbitrary deontic Restall frame into a deontic matrix frame.

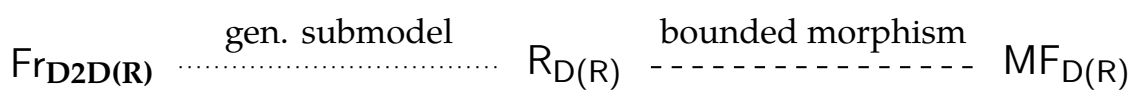

We start with some facts about D2D, which are all left as an exercise to the reader:

Fact 1. The following are all provable in 2D (Fusco, 2020b):
$X \quad \dagger(@ \leftrightarrow \leftrightarrow \phi)$
$\operatorname{Red}_{\dagger} \dagger \dagger \phi \leftrightarrow \dagger \phi$
$Y @(\dagger \phi \leftrightarrow \phi)$
$\operatorname{Red}_{@} @ \phi \leftrightarrow \leftrightarrow \phi$
$\dagger \diamond \dagger \quad \dagger \diamond \dagger \phi \leftrightarrow \diamond \dagger \phi$
$A @ A \phi \rightarrow @ \phi$

In addition, the following is provable in $\mathbf{D} 2 \mathrm{D}$ :

@5 $\hat{O} \quad \hat{O} @ \phi \rightarrow @ \phi$

Definition 12. A Kripke frame is a tuple $\mathcal{F}=\left\langle W, R_{\square}, R_{\Theta}, R_{\dagger}, R_{O}\right\rangle$ where $W$ is a set of points and each $R_{\triangle}$ is a binary relation on $W$.

Fact 2. The axioms of D2D(R) have the following (global) first-order correspondents in Kripke frames:
$T_{\square} \quad R_{\square}$ is reflexive
$G \quad R_{@} \subseteq R_{\square}$
$5_{\square} \quad R_{\square}$ is Euclidean
$R_{@} \quad R_{@}$ is a function
$@ 5 \diamond \quad \forall w, v, u:$
$\quad\left(w R_{\square} v \& w R_{@} u\right) \Rightarrow v R_{\circledast} u$
$R_{\dagger} \quad R_{\dagger}$ is a function
$X \rightarrow \forall w, v, u:$

the sense of Blackburn et al. 2002, p. 206. However, the same is not true for the proof of Theorem 4 in $\S \mathrm{B}$ (since $R_{O}^{\cup}$ may not satisfy the appropriate constraints). 


$$
\begin{aligned}
& \left(w R_{\dagger} v \& v R_{@} u\right) \Rightarrow v=u \quad Y_{\rightarrow} \forall w, v, u: \\
& \left(w R_{@} v \& v R_{\dagger} u\right) \Rightarrow v=u \\
& 4_{A} \quad R_{\square} \circ R_{\dagger} \text { is transitive } \\
& 5_{A} \quad \forall x, y, a, b: \\
& \left(x R_{\square} y \& x R_{\square} a \& a R_{\dagger} b\right) \Rightarrow \\
& \exists c\left(b R_{\square} c \& \forall d\left(c R_{\dagger} d \Rightarrow y R_{\dagger} d\right)\right) \\
& M C \quad R_{O} \subseteq R_{\square} \\
& D_{O} \quad R_{O} \text { is serial } \\
& U_{O} \quad R_{O} \text { is shift-reflexive } \\
& \text { DR } \forall w, v, u \text { : } \\
& \left(w R_{\square} v \& w R_{O} u\right) \Rightarrow v R_{O} u
\end{aligned}
$$

The correspondents for $X$ and $Y$ in Fact 1 are as follows:

$X \quad$ Within $\operatorname{Im} g\left(R_{\dagger}\right), R_{@}$ is the identity relation

$Y$ Within $\operatorname{Im} g\left(R_{\circledast}\right), R_{\dagger}$ is the identity relation

The following facts hold of any D2D-frame:

Fact 3. $\operatorname{Im} g\left(R_{\Theta}\right)=\operatorname{Im} g\left(R_{\dagger}\right)$.

Fact 4. $\forall d_{1}, d_{2} \in \operatorname{Im} g\left(R_{\Theta}\right):$ if $d_{1}\left(R_{\square} \circ R_{\dagger}\right) d_{2}$, then $d_{2}\left(R_{\square} \circ R_{\dagger}\right) d_{1}$.

Fact 5. $R_{\square} \circ R_{\dagger}$ an equivalence relation on $\operatorname{Img}\left(R_{\circledast}\right)$.

Fact 6. Suppose $w R_{\circledast} d_{1}$ and $w R_{\dagger} d_{2}$. Then $\exists w^{\prime}$ s.t. $w^{\prime} R_{\circledast} d_{2}$ and $w^{\prime} R_{\dagger} d_{1}$.

Before we show $\operatorname{Fr}_{D 2 D(R)}$ is modally equivalent to $R_{D(R)}$, it will help to prove the following lemma. (Notation: if $R_{\triangle}$ is a function, we use " $R_{\triangle}(x)$ " for the unique $y$ such that $x R_{\triangle} y$ )

Lemma 1. For any point-generated subframe $\mathcal{F}_{w}=\left\langle W^{\prime}, R_{\square}^{\prime}, R_{\dagger}^{\prime}, R_{\mathbb{Q}^{\prime}}^{\prime}, R_{O}^{\prime}\right\rangle \in \mathrm{Fr}_{\mathbf{D} 2 \mathrm{D}}$ : $W^{\prime}=R_{\square}\left[R_{\dagger}\left[R_{\square}[\{w\}]\right]\right]$.

Proof. We let $X:=R_{\square}\left[R_{\dagger}\left[R_{\square}[\{w\}]\right]\right]$. It suffices to show (1) $w \in X$ and (2) that for each operator $\nabla$ that $R_{\nabla}[X] \subseteq X$. These are proven in Fusco 2020b except for the $O$ case for (2), which is easy since $R_{O} \subseteq R_{\square}$ and we have $T_{\square}$.

Definition 13. A (rigid) deontic Restall Frame is a frame $\mathcal{R}=\left\langle W, R_{\square}, R_{\Theta}, R_{\dagger}, R_{O}\right\rangle$ such that 
1. $R_{\square}$ is an equivalence relation

2. $R_{@}$ is a function such that

(a) $w R_{@} v \rightarrow w R_{\square} v$

(b) $R_{@}$ maps any two $R_{\square}$-related worlds to the same point

3. $R_{\dagger}$ is a function such that:

(a) for any $w: R_{\dagger}\left[R_{\square}[\{w\}]\right]=\operatorname{Img}\left(R_{\circledast}\right)$

(b) $R_{\dagger}$ is reflexive over $\operatorname{Im} g\left(R_{\circledast}\right)$

4. $R_{O}$ is a (rigid,) serial, shift-reflexive subrelation of $R_{\square}$.

Let $R_{D(R)}$ be the class of (rigid) deontic Restall frames.

Lemma 2. $R_{D(R)} \subset \operatorname{Fr}_{D 2 D(R)}$.

Proof. To verify this, it suffices to go through the first-order correspondents of D2D(R) from Fact 2.

Lemma 3. Every point-generated subframe $\mathcal{F}_{w} \in \operatorname{Fr}_{\mathbf{D} 2 \mathbf{D}(\mathbf{R})}$ is a (rigid) deontic Restall frame.

Proof. Where $\mathcal{F}_{w}=\left\langle W^{\prime}, R_{\Theta}^{\prime}, R_{\dagger}^{\prime}, R_{\square}^{\prime}, R_{O}^{\prime}\right\rangle$ is a $w$-generated subframe in $\operatorname{Fr}_{\mathbf{D} 2 \mathrm{D}}$, let $v$ be an arbitrarily chosen world in $W^{\prime}$. We want to establish that $\mathcal{F}_{w}$ satisfies conditions 1-4 of deontic Restall frames. Conditions 1-3 are established in Fusco 2020b. Condition 4 follows from the correspondences in Fact 2.

Theorem 10. $\operatorname{Fr}_{\mathrm{D} 2 \mathrm{D}(\mathrm{R})}$ and $\mathrm{R}_{\mathrm{D}(\mathrm{R})}$ are modally equivalent.

Proof. By Lemma 2, any formula falsifiable in $\mathcal{R} \in R_{D(R)}$ is falsifiable in some $\mathcal{F} \in \operatorname{Fr}_{\text {D2D(R) }}$. By Lemma 3, any formula falsifiable in a point-generated subframe $\mathcal{F}_{w} \in \operatorname{Fr}_{\mathbf{D} 2 \mathrm{D}(\mathrm{R})}$ is falsifiable in some $\mathcal{R} \in \mathrm{R}_{\mathrm{D}(\mathrm{R})}$. But any formula falsifiable in a $\mathcal{F} \in \operatorname{Fr}_{\mathbf{D} 2 \mathbf{D}(\mathbf{R})}$ is falsifiable in a point-generated subframe (Blackburn et al., 2002, Proposition 2.6.).

Now we just need to reduce $R_{D(R)}$ to $M F_{D(R)}$. Some notation:

- We write $R[Y]$ for the image of a set $Y$ under $R$.

- Let $D=\operatorname{Im} g\left(R_{\Theta}\right)$. We will use $i, j \ldots$ as indices over $D$.

- Where $\mathcal{R}$ is a deontic Restall frame, let $C$ be the set of $R_{\square}$-cells in $\mathcal{R}$. Since there is one $d \in D$ in each such cell, $C$ is the cardinality of $D$. 
Fact 7. $\cup_{i \in D} c_{i}=W$.

Fact 8. There is a unique $R_{\Theta}$-fixed point in each $R_{\square}$-cell $c_{i} \subset W$.

Notation: call this $R_{\circledast}$-fixed point $R_{\circledast}\left(c_{i}\right)$.

Fact 9. For any $w$ and any $c_{j} \subseteq W, \exists ! v \in c_{j}$ s.t. $w R_{\dagger} v$.

More notation: where $c_{i} \in C$, let $c_{i}^{j}$ be the set $\left\{w \in c_{i} \mid \exists v \in c_{j}: w R_{\dagger} v\right\}$. Let $R_{\dagger}\left(c_{i}^{j}\right)$ be the unique $v \in c_{j}$ such that $\forall w \in c_{i}^{j}, w R_{\dagger} v$. That $R_{\dagger}\left(c_{i}^{j}\right)$ is a fixed point of $R_{\dagger}$ follows from $\operatorname{Red}_{\dagger}$.

Corollary 1. Each $c_{i}$ can be partitioned into $\left\{c_{i}^{j} \mid j \in D\right\}$, where $c_{i}^{j}$ is a nonempty subset of $c_{i}$ such that $\forall w \in c_{i}^{j}, w R_{\dagger}\left(R_{\Theta}\left(c_{j}\right)\right)$. Hence $\bigcup_{j \in D} c_{i}^{j}=c_{i}$, and $\bigcup_{i \in D}\left(\bigcup_{j \in D} c_{i}^{j}\right)=$ W.

Theorem 11. $R_{D(R)}$ and $M F_{D(R)}$ are modally equivalent.

Proof. Since $M F_{D(R)} \subset R_{D(R)}$, every (rigid) deontic matrix frame is equivalent to some (rigid) deontic Restall frame. For the converse, given a deontic Restall frame $\mathcal{R}$ with domain $W$ and $\operatorname{Im} g\left(R_{\circledast}\right)=D$, we will build a matrix frame $\mathcal{M}$ and construct, row-by-row, a surjective bounded morphism $f$ from $\mathcal{M}$ to $\mathcal{R}$, from which modal equivalence follows (Blackburn et al., 2002, Proposition 2.14).

The points of our matrix frame $\mathcal{M}$ will be $(D \times W) \times(D \times W)$. For any $i, j \in D$ and $y \in W$, let $A_{i, y}^{j}$ be the set $\{\langle i, y\rangle\} \times\{\langle j, x\rangle: x \in W\}$. Note that $\left|A_{i, y}^{j}\right|=|W|$.

For each $i, j \in D$ and $y \in W$, fix some surjective $g_{i, y}^{j}: W \rightarrow c_{i}^{j}$ such that if $i=j$ and $y=x$, then $g_{i, y}^{j}(x)=R_{\Theta}\left(c_{i}\right)$. (This condition maps the "diagonal" points of the matrix frame into $D$ in the deontic Restall frame.) We know such surjective functions exist since $\left|A_{i, y}^{j}\right| \geqslant\left|c_{i}^{j}\right|$.

The accessibility relations $R_{\square}^{\mathcal{M}}, R_{\Theta}^{\mathcal{M}}$, and $R_{\dagger}^{\mathcal{M}}$ are already determined by the definition of a deontic matrix frame. To define $R_{O}^{\mathcal{M}}$, first define a function $F:(D \times$ $W) \times(D \times W) \rightarrow W$ as follows: $F(\langle\langle i, y\rangle,\langle j, x\rangle\rangle)=g_{i, y}^{j}(x)$. We define $R_{O}^{\mathcal{M}}$ in terms of $F$ :

$$
\langle\langle i, y\rangle,\langle j, x\rangle\rangle R_{O}^{\mathcal{M}}\left\langle\langle i, y\rangle,\left\langle j^{\prime}, x^{\prime}\right\rangle\right\rangle \Leftrightarrow F(\langle\langle i, y\rangle,\langle j, x\rangle\rangle) R_{O} F\left(\left\langle\langle i, y\rangle,\left\langle j^{\prime}, x^{\prime}\right\rangle\right\rangle\right) .
$$

We will now show that $F$ is a bounded morphism from $\mathcal{M}$ to $\mathcal{R} .{ }^{34}$ To do this, it

${ }^{34} \mathrm{~F}$ is a bounded morphism if it satisfies the following conditions for each $\triangle \in\{\square, @, \dagger, O\}$ (Blackburn et al., 2002, pg. 59):

1. $w$ and $F(w)$ satisfy the same proposition letters;

2. if $w R_{\triangle}^{\mathcal{M}} v$ then $F(w) R_{\triangle} F(v)$ (the Forth condition);

3. if $F(w) R_{\triangle} v^{\prime}$ then there exists some $v$ such that $w R_{\triangle}^{\mathcal{M}} v$ and $F(v)=v^{\prime}$ (the Back condition). 
suffices to go through the Back and Forth conditions for $\triangle \in\{\square, @, \dagger, O\}$ for each point. The only new case not in the proof from Fusco 2020b is the $O$ case.

(O, Forth) Immediate by the definition of $R_{O}^{\mathcal{M}}$.

$\left(O\right.$, Back) Suppose $F(\langle\langle i, y\rangle,\langle j, x\rangle\rangle) R_{O} v^{\prime}$. We want to show that there is some $\left\langle\langle i, y\rangle,\left\langle j^{\prime}, x^{\prime}\right\rangle\right\rangle$ such that $\langle\langle i, y\rangle,\langle j, x\rangle\rangle R_{O}^{X}\left\langle\langle i, y\rangle,\left\langle j^{\prime}, x^{\prime}\right\rangle\right\rangle$ and also that $F\left(\left\langle\langle i, y\rangle,\left\langle j^{\prime}, x^{\prime}\right\rangle\right\rangle\right)=v^{\prime}$. To witness this existential, we can choose any $\left\langle\langle i, y\rangle,\left\langle j^{\prime}, x^{\prime}\right\rangle\right\rangle$ such that $F\left(\left\langle\langle i, y\rangle,\left\langle j^{\prime}, x^{\prime}\right\rangle\right\rangle\right)=v^{\prime}$ (we know one exists since $F$ is surjective).

Hence, $F$ is a bounded morphism, and so $\mathcal{M}$ is modally equivalent to $\mathcal{R}$.

\section{B Axiomatizing Deontic Åqvist Logic}

In this appendix, we prove Theorem 4: $\mathbf{F}$ is sound and complete for the class of Åqvist uniform deontic matrix models DMÅU. (The proof that FR is sound and complete for DMÅUR is analogous.) Soundness is straightforward and left to the reader. For completeness, the strategy will be to bootstrap off the completeness result for D2D in § A.

First, we need to prove some lemmas about the expressive power of D2D.

Lemma 4. For any $\mathcal{L}^{\mathrm{D} 2 \mathrm{D}}$-formula $\phi$, the following formulas are strictly valid over DM:

(a) $\square(@ \phi \vee \psi) \leftrightarrow(@ \phi \vee \square \psi)$

(b) $O(@ \phi \vee \psi) \leftrightarrow(@ \phi \vee O \psi)$.

Recall the definition of explicitly 1D formulas (Definition 10).

Lemma 5. For any deontic matrix model $\mathcal{M}=\left\langle W \times W, R_{\square}, R_{囚}, R_{\dagger}, R_{O}, V\right\rangle$, if $\mathcal{M}$ satisfies $\AA$ and $U$ everywhere, then for any $w, w^{\prime}, v \in W$, any explicitly 1D $\mathcal{L}^{\text {D2D }}$-formula $\phi$ :

$$
\mathcal{M}, w, v \Vdash \phi \quad \Leftrightarrow \quad \mathcal{M}, w^{\prime}, v \Vdash \phi .
$$

Proof. By induction.

Definition 14. An @-atom is a $\mathcal{L}^{\mathrm{D} 2 \mathrm{D}}$-formula that is either explicitly $1 \mathrm{D}$, or is of the form @ $\phi$ where $\phi$ is explicitly 1D.

The following is an extension of a result for languages without $\dagger$ or $O$ proven in Hazen et al. 2013: 
Lemma 6. Every $\mathcal{L}^{\text {D2D }}$-formula is strictly equivalent to a boolean combination of $@$-atoms over DMÅU.

Proof. By induction on the structure of $\mathcal{L}^{\mathrm{D} 2 \mathrm{D}}$-formulas. Throughout, we'll use " $\phi \equiv \psi$ " to mean $\phi$ and $\psi$ are strictly equivalent over DMÅU. The atomic and boolean cases are trivial. We'll present the other cases. Assume for inductive hypothesis that the claim holds of $\phi$. In particular, assume that:

$$
\phi \equiv \bigwedge_{i=1}^{k} \bigvee_{j=1}^{n_{i}} \alpha_{i, j}
$$

where each $\alpha_{i, j}$ is an @-atom.

( $\square$ ) Since $\square$ commutes with conjunction:

$$
\square \phi \equiv \square \bigwedge_{i=1}^{k} \bigvee_{j=1}^{n_{i}} \alpha_{i, j} \equiv \bigwedge_{i=1}^{k} \square \bigvee_{j=1}^{n_{i}} \alpha_{i, j}
$$

So it suffices to show that each $\square \bigvee_{j=1}^{n_{i}} \alpha_{i, j}$ is equivalent to a boolean combination of @-atoms. First, write $\bigvee_{j=1}^{n_{i}} \alpha_{i, j}$ as:

$$
@ \beta_{i, 1} \vee \cdots \vee @ \beta_{i, m} \vee \gamma_{i, m+1} \vee \cdots \vee \gamma_{i, j}
$$

where each $\beta_{i, x}$ and $\gamma_{i, y}$ are explicitly 1D. Then by Lemma 4(a):

$$
\begin{aligned}
\square \bigvee_{j=1}^{n_{i}} \alpha_{i, j} & \equiv \square\left(@ \beta_{i, 1} \vee \cdots \vee @ \beta_{i, m} \vee \gamma_{i, m+1} \vee \cdots \vee \gamma_{i, j}\right) \\
& \equiv @ \beta_{i, 1} \vee \cdots \vee @ \beta_{i, m} \vee \square\left(\gamma_{i, m+1} \vee \cdots \vee \gamma_{i, j}\right)
\end{aligned}
$$

But now all these terms are @-atoms.

$(O)$ Similar to the ( $\square)$ case.

(@) Since @ commutes with booleans:

$$
@ \phi \equiv @ \bigwedge_{i=1}^{k} \bigvee_{j=1}^{n_{i}} \alpha_{i, j} \equiv \bigwedge_{i=1}^{k} \bigvee_{j=1}^{n_{i}} @ \alpha_{i, j}
$$

Now, either $\alpha_{i, j}$ is explicitly 1D, in which case @ $\alpha_{i, j}$ is an @-atom, or $\alpha_{i, j}=$ $@ \beta_{i, j}$ where $\beta_{i, j}$ is explicitly $1 \mathrm{D}$, in which case @ $\alpha_{i, j} \equiv @ @ \beta_{i, j} \equiv @ \beta_{i, j} \equiv$ $\alpha_{i, j}$, so we can replace @ $\alpha_{i, j}$ with $\alpha_{i, j}$. The result is therefore a boolean combination of @-atoms. 
$(\dagger)$ Since $\dagger$ commutes with booleans:

$$
\dagger \phi \equiv \dagger \bigwedge_{i=1}^{k} \bigvee_{j=1}^{n_{i}} \alpha_{i, j} \equiv \bigwedge_{i=1}^{k} \bigvee_{j=1}^{n_{i}} \dagger \alpha_{i, j}
$$

Now, either $\alpha_{i, j}$ is explicitly 1D or $\alpha_{i, j}=@ \beta_{i, j}$ where $\beta_{i, j}$ is explicitly 1D. In the former case, $\uparrow \alpha_{i, j} \equiv \alpha_{i, j}$ by Lemma 5 . In the latter case, $\uparrow \alpha_{i, j} \equiv \dagger @ \beta_{i, j} \equiv \dagger \beta_{i, j}$, which is equivalent to $\beta_{i, j}$ by Lemma 5 again. So either way, $\uparrow \alpha_{i, j} \equiv \alpha_{i, j}$, which means $\phi$ is strictly equivalent to $\dagger \phi$ already.

Theorem 12. F is sound and complete for DMÅU.

Proof. Let $\Gamma$ be a F-consistent set of formulas. Take a maximal F-consistent extension $\Gamma^{+} \supseteq \Gamma$ (the proof that one exists is standard). By Theorem 3 , there is a deontic matrix model $\mathcal{M}$ and some $y, x \in W$ such that $\mathcal{M}, y, x \Vdash \Gamma^{+}$. We first show that the valuation $V$ is already Aqvist. Then we show how to transform $\mathcal{M}$ into an equivalent model whose deontic accessibility relation is uniform.

First, $V$ is Åqvist: since $\square \dagger \square(p \leftrightarrow \dagger p) \in \Gamma^{+}$for all $p \in$ Prop, it follows that $\mathcal{M}, w, v \Vdash p \leftrightarrow \dagger p$ for all $w, v \in X$. Henec, for all $w, w^{\prime}, v \in X$ :

$$
\begin{aligned}
\langle w, v\rangle \in V(p) & \Leftrightarrow \mathcal{M}, w, v \Vdash p \\
& \Leftrightarrow \mathcal{M}, w, v \Vdash \dagger p \\
& \Leftrightarrow \mathcal{M}, v, v \Vdash p \\
& \Leftrightarrow \mathcal{M}, w^{\prime}, v \Vdash \dagger p \\
& \Leftrightarrow \mathcal{M}, w^{\prime}, v \Vdash p \\
& \Leftrightarrow\left\langle w^{\prime}, v\right\rangle \in V(p) .
\end{aligned}
$$

Next, we show how to transform $\mathcal{M}$ into an equivalent one whose deontic accessibility relation is uniform. Define $R_{O}^{\cup}$ as follows:

$$
\langle w, v\rangle R_{O}^{\cup}\langle w, u\rangle \Leftrightarrow \quad \exists z:\langle z, v\rangle R_{O}\langle z, u\rangle
$$

Define $\mathcal{M}^{\cup}=\left\langle W \times W, R_{\square}, R_{囚}, R_{\dagger}, R_{O}^{\cup}, V\right\rangle$. Clearly, $R_{O}^{\cup}$ is uniform, serial, and shift-reflexive. (Note also that if $R_{O}$ is rigid, so is $R_{O}^{\cup}$.)

Lemma 7. For all $w, v \in W$ and all explicitly $1 \mathrm{D} \mathcal{L}^{\mathrm{D} 2 \mathrm{D}}$-formulas $\phi$ :

$$
\mathcal{M}, w, v \Vdash \phi \quad \Leftrightarrow \quad \mathcal{M}^{\cup}, w, v \Vdash \phi .
$$

Proof. By induction on the complexity of $\phi$. We only present the $O$ case (the others are straightforward). Suppose for inductive hypothesis that the claim holds of $\phi$. 
Clearly, if $\mathcal{M}^{\cup}, w, v \Vdash O \phi$, then $\mathcal{M}, w, v \Vdash O \phi$ since $R_{O} \subseteq R_{O}^{\cup}$. So we just need to establish the converse.

Suppose $\mathcal{M}^{\cup}, w, v \forall-O \phi$. Thus, for some $u \in W$, we have $\langle w, v\rangle R_{O}^{\cup}\langle w, u\rangle$ and $\mathcal{M}^{\cup}, w, u \Downarrow \psi \phi$. By definition of $R_{O}^{\cup}$, for some $z \in W,\langle z, v\rangle R_{O}\langle z, u\rangle$. And by inductive hypothesis, $\mathcal{M}, w, u \Vdash \psi \phi$. Hence, by Lemma $5, \mathcal{M}, z, u \Downarrow \phi$. So $\mathcal{M}, z, v \Downarrow \mathcal{O} \phi$. But again by Lemma $5, \mathcal{M}, w, v \Downarrow O \phi$.

Lemma 8. For all $w, v \in W$ and all @-atoms $\phi$ :

$$
\mathcal{M}, w, v \Vdash \phi \quad \Leftrightarrow \quad \mathcal{M}^{\cup}, w, v \Vdash \phi .
$$

Proof. If $\phi$ is an explicitly $1 \mathrm{D} \mathcal{L}^{\mathrm{D} 2 \mathrm{D}}$-formula, then this is ensured by Lemma 7. If $\phi=@ \psi$ where $\psi$ is explicitly $1 \mathrm{D}$, then:

$$
\begin{aligned}
\mathcal{M}, w, v \Vdash @ \psi & \Leftrightarrow \mathcal{M}, w, w \Vdash \psi \\
& \Leftrightarrow \mathcal{M}^{\cup}, w, w \Vdash \psi \text { (Lemma 7) } \\
& \Leftrightarrow \mathcal{M}^{\cup}, w, v \Vdash @ \psi .
\end{aligned}
$$

Hence, by Lemma 6, for any $\mathcal{L}^{\mathbf{D} 2 \mathrm{D}}$-formula $\phi$ and any $w, v \in W: \mathcal{M}, w, v \Vdash \phi$ iff $\mathcal{M}^{\cup}, w, v \Vdash \phi$. So $\mathcal{M}^{\cup}, y, x \Vdash \Gamma^{+}$.

Note that one cannot axiomatize consequence over DMU simply by dropping $\AA$ from the axiomatization. The inductive step for $O$ in the proof of Lemma 7 relies on Lemma 5, which in turn relies on the $\AA$ axiom for the base case. This use of $\AA$ is ineliminable: for $U$ is not valid over the general class of (even rigid) uniform deontic matrix models. A counterexample is given in Figure 11.

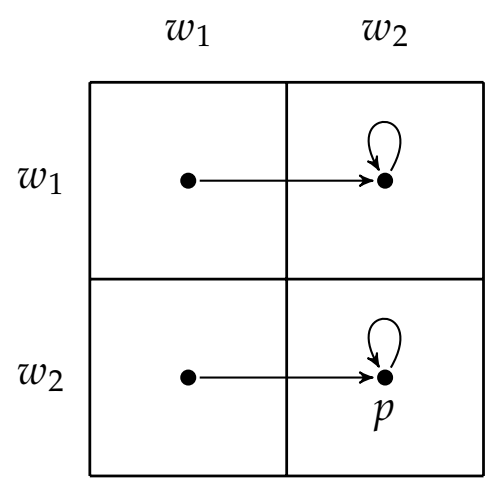

Figure 11: A rigid, uniform deontic matrix model that does not satisfy $O p \leftrightarrow \dagger O p$ at $\left\langle w_{1}, w_{2}\right\rangle$. 


\section{References}

Anderson, Alan Ross. 1958. "A Reduction of Deontic Logic to Alethic Modal Logic." Mind 67:100-103.

Åqvist, Lennart. 1973. "Modal Logic with Subjunctive Conditionals and Dispositional Predicates." Journal of Philosophical Logic 2:1-76.

Barker, Chris. 2010. "Free choice permission as resource-sensitive reasoning." Semantics and Pragmatics 3:1-38.

Blackburn, Patrick, de Rijke, Maarten, and Venema, Yde. 2002. Modal Logic. Cambridge University Press.

Bledin, Justin. 2015. “Modus Ponens Defended." Journal of Philosophy 112:57-83.

Burgess, John. 1999. "Which modal logic is the right one?" Notre Dame Journal of Formal Logic 40:81-93.

Carr, Jennifer. 2014. “The If P, Ought P Problem." Pacific Philosophical Quarterly 95:555-583.

Chalmers, David. 2004. "Epistemic two-dimensional semantics." Philosophical Studies 118:153-226.

—. 2012. Constructing the World. Oxford University Press.

Crossley, John and Humberstone, Lloyd. 1977. "The Logic of 'actually'." Reports on Mathematical Logic 8:11-29.

Davies, Martin and Humberstone, Lloyd. 1980. “Two Notions of Necessity.” Philosophical Studies 38:1-30.

Dayal, Veneeta. 1996. Locality in Wh-quantification: Questions and Relative Clauses in Hindi. Dordrecht: Kluwer Academic Publishers.

—. 2016. Questions. Oxford University Press.

Fox, Danny. 2007. "Free Choice Disjunction and the Theory of Scalar Implicatures." In U. Sauerland and P. Stateva (eds.), Presupposition and Implicature in Compositional Semantics. New York, Palgrave Macmillan.

Fritz, Peter. 2014. "What is the Correct Logic of Necessity, Actuality and Apriority?" Review of Symbolic Logic 7:385-414.

Fusco, Melissa. 2015. "Deontic Modality and the Semantics of Choice." Philosophers' Imprint 15:1-27. 
—. 2019. "Naturalizing Deontic Logic: Indeterminacy, Diagonalization, and SelfAffirmation." Philosophical Perspectives 32:165-187.

- 2020a. "Free choice effects and exclusive disjunction." Inquiry 1-15. doi: 10.1080/0020174X.2020.1758768.

-. 2020b. "A Two-Dimensional Logic for Diagonalization and the A Priori." Synthese 1-16. doi:10.1007/s11229-020-02574-7.

Gregory, Dominic. 2001. "Completeness and Decidability Results for Some Propositional Modal Logics Containing "Actually" Operators." Journal of Philosophical Logic 30:57-78.

Groenendijk, Jeroen and Stokhof, Martin. 1982. "Semantic Analysis of WhComplements." Linguistics and Philosophy 5:172-233.

Hare, Caspar and Hedden, Brian. 2016. "Self-Reinforcing and Self-Frustrating Decisions." Noûs 50:604-628.

Hawthorne, John and Magidor, Ofra. 2009. "Assertion, Context, and Epistemic Accessibility." Mind 118.

Hazen, Allen P., Rin, Benjamin G., and Wehmeier, Kai F. 2013. "Actuality in Propositional Modal Logic." Studia Logica 101:487-503.

Heim, Irene. 2004. "Lecture notes on indexicality." Notes for class taught at MIT .

Holliday, Wesley, Icard, Thomas, and Hoshi, Tomohiro. 2013. "Information Dynamics and Uniform Substitution." Synthese 190:31-55.

Humberstone, Lloyd. 2004. "Two-Dimensional Adventures." Philosophical Studies 118:17-65.

-. 2020. "Sentence Connectives in Formal Logic." The Stanford Encyclopedia of Philosophy https://plato.stanford.edu/archives/spr2020/entries/ connectives-logic/.

Kamp, Hans. 1971. “Formal Properties of 'Now'." Theoria 377:227-273.

—. 1973. "Free Choice Permission." Proceedings of the Aristotelian Society, New Series 74:57-74.

Kanger, Stig. 1971. "New Foundations for Ethical Theory." In Risto Hilpinen (ed.), Deontic Logic: Introductory and Systematic Readings. Dordrecht: D. Reidel.

Kaplan, David. 1989. “Demonstratives." In Joseph Almog, John Perry, and Howard Wettstein (eds.), Themes from Kaplan. Oxford University Press. 
Kaufmann, Magdalena. 2016. "Free choice is a form of dependence." Natural Language Semantics 24:247-290.

Kratzer, Angelika. 1977. "What must and can must and can mean." Linguistics and Philosophy 1:337-355.

—. 1981. "The Notional Category of Modality." In Hans-Jürgen Eikmeyer and Hannes Rieser (eds.), Words, Worlds, and Context. de Gruyter.

—. 1986. "Conditionals." Chicago Linguistics Society 22:1-15.

Kripke, Saul. 1980. Naming and Necessity. Harvard University Press.

Lewis, David. 1973. Counterfactuals. Oxford: Blackwell.

—. 1982. "“Whether" Report." In Tom Pauli (ed.), Philosophical Essays Dedicated to Lennart Åqvist on His Fiftieth Birthday, 194-206. University of Uppsala Press.

MacFarlane, John and Kolodny, Niko. 2010. "Ifs and Oughts." Journal of Philosophy 107:115-143.

McNamara, Paul. 2010. "Deontic Logic." Stanford Encyclopedia of Philosophy http: //plato.stanford.edu/archives/fall2010/entries/logic-deontic/.

Meredith, C. A. and Prior, A. N. 1965. "Modal Logic with Functorial Variables and a Contingent Constant." Notre Dame Journal of Formal Logic 6:99-109.

Parfit, Derek. 1984. Reasons and Persons. Oxford University Press.

Paul, L. A. 2014. Transformative Experience. Oxford University Press.

-. 2015. "What You Can't Expect When You're Expecting." Res Philosophica 92:149-170.

Roelofsen, Floris. 2019. "Semantic Theories of Questions." In Oxford Research Encyclopedia of Linguistics. Oxford University Press.

Ross, Alf. 1941. "Imperatives in Logic." Theoria 7:53-71.

Segerberg, Krister. 1973. "Two-Dimensional Modal Logic." Journal of Philosophical Logic 2:77-96.

Simons, Mandy. 2005. "Dividing Things Up: The Semantics of Or and the Modal/Or Interaction." Natural Language Semantics 13:271-316.

Smiley, Timothy. 1982. "The Schematic Fallacy." Proceedings of the Aristotelian Society 83:1-17. 
Stalnaker, Robert. 1975. "Indicative Conditionals." In Context and Content. Oxford University Press. In Stalnaker (1999).

-. 1978. "Assertion." In Context and Content, 78-95. Oxford University Press. In Stalnaker (1999).

—. 1981. "Indexical Belief." Synthese 49:129-151.

-. 1999. Context and Content. Oxford, Oxford University Press.

van Fraassen, Bas. 1977. "The Only Necessity is Verbal Necessity." Journal of Philosophy 74:71-85.

Veltman, Frank. 1996. "Defaults in Update Semantics." Journal of Philosophical Logic 25:221-261.

Vlach, Frank. 1973. 'Now' and 'Then': A Formal Study in the Logic of Tense Anaphora. Ph.D. thesis, UCLA.

Williamson, Timothy. 2013. Modal Logic as Metaphysics. Oxford University Press.

Yalcin, Seth. 2007. “Epistemic Modals.” Mind 116:983-1026.

—. 2015. "Actually, Actually." Analysis 75:185-191. 